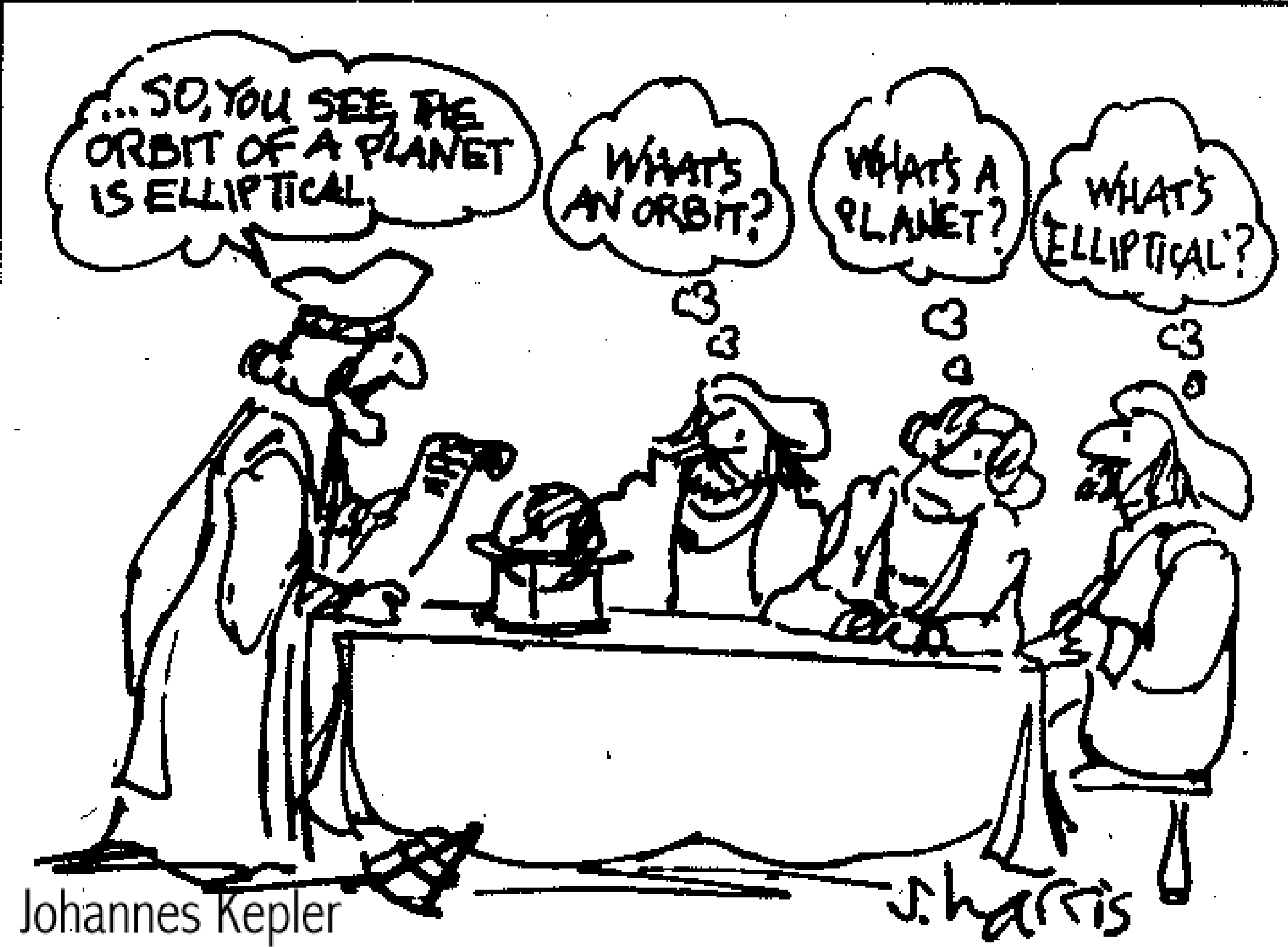

frank@grupp-astro.de

Frank Krupp - Spectral synthesis basics

Slide 1 


\section{Outline of the lecture}

I. Who I am, what I do.

II.Cool star element abundances

I. Why do we want to know

II.What do we want to know

III.How do we find out

I. The general framework

II.Line formation in model atmospheres

III. The curve of growth 


\section{How things started ...}

- ... my first spectrograph!

- 1987 as a school project

- Objective prism + normal camera

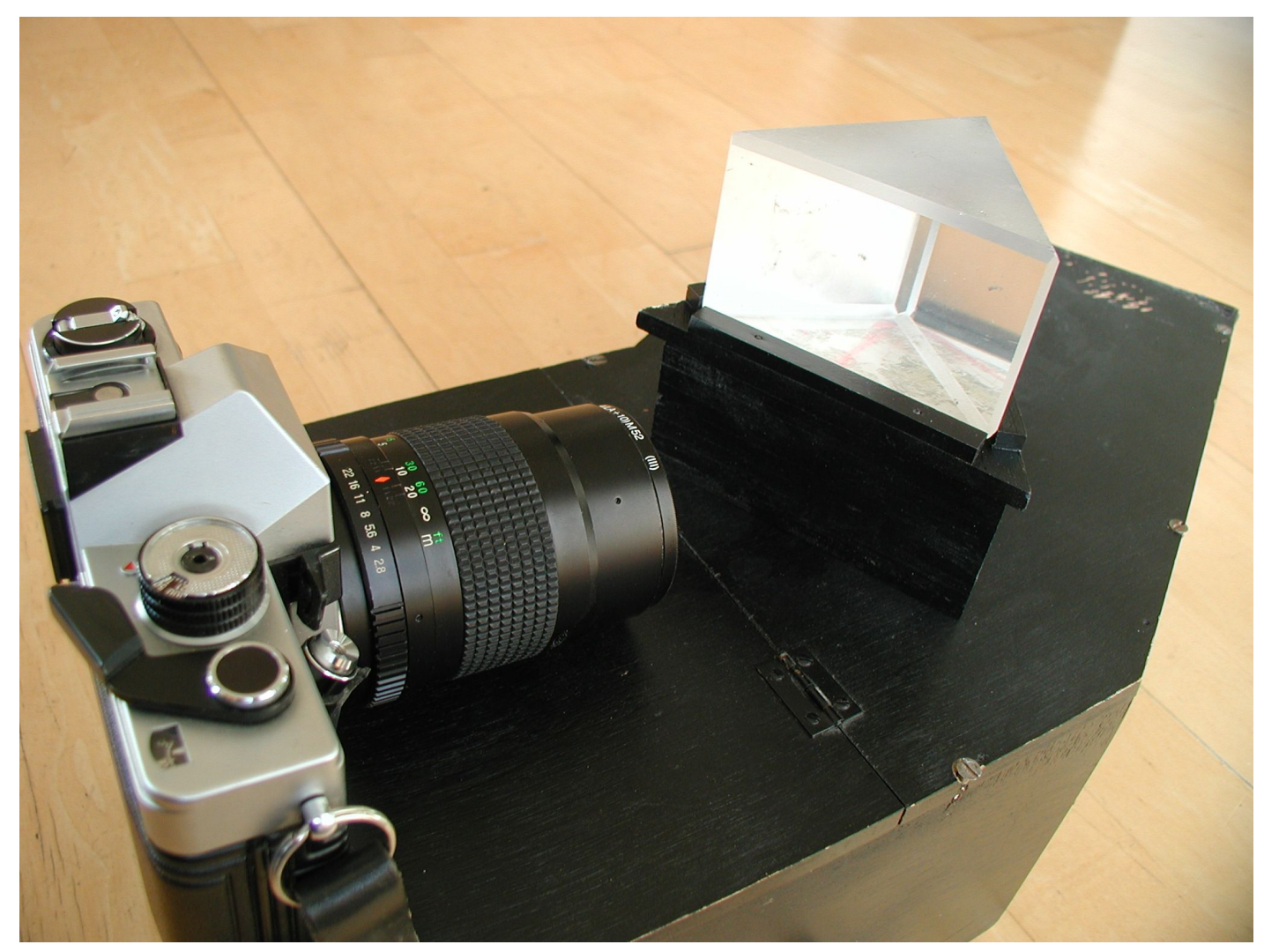




\section{Instrument development (1)}

-FOCES a R=64000 Echelle spectrograph

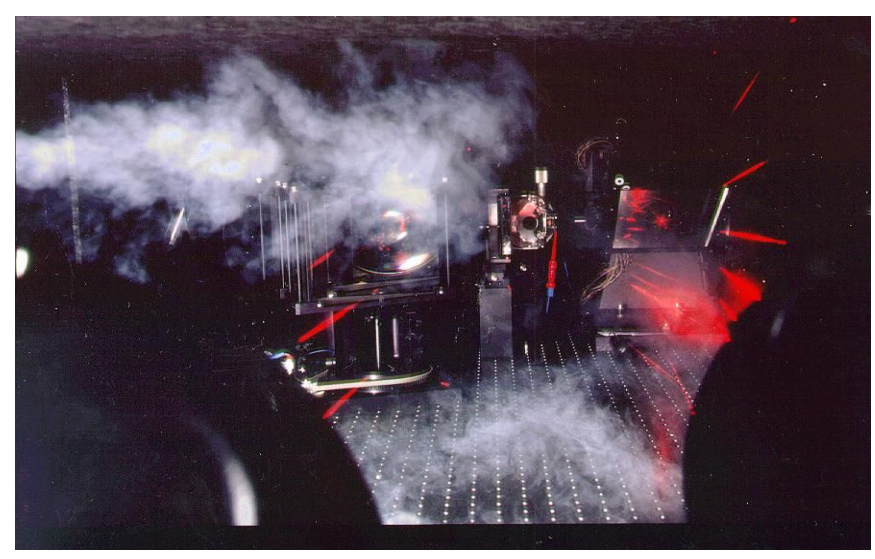

-Fraunhofer Telescope Wendelstein (2m)

-Optical design (with Kayser-Threde Munich)

-Instruments for the telescope

-WFI - wide field imager

-3KK - three channel camera (2Vis+1IR)

-Virus-W - 270 channel spectrograph

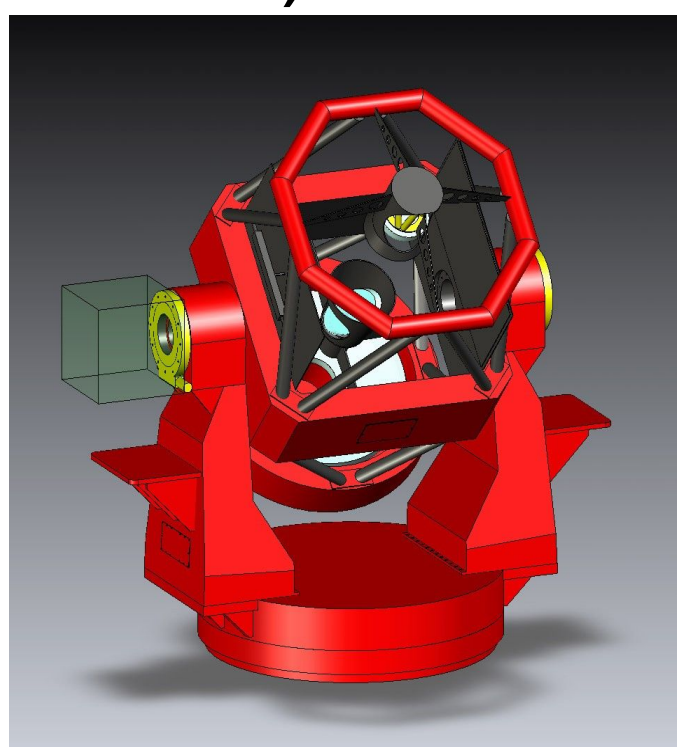




\section{Scientific interests}

- Stellar atmosphere models

- Opacity sampling

- Background opacities

- Convection in stars

- Precise stellar parameters

- Line formation

- Model improvements 


\section{Outline of the lecture}

I. Who I am, what I do.

II.Cool star element abundances

I. Why do we want to know

II.What do we want to know

III. How do we find out

I. The general framework

II.Line formation in model atmospheres

III.The curve of growt 


\section{Element abundances (1)}

- In the

- Hydr

- Stellar New born stars ejected

- Re-eje

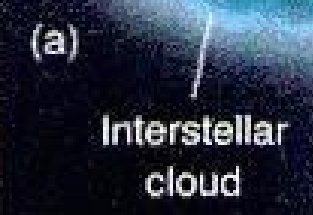

- Stella

- Plan

- Supe

(b)

(c)

Expanding waves

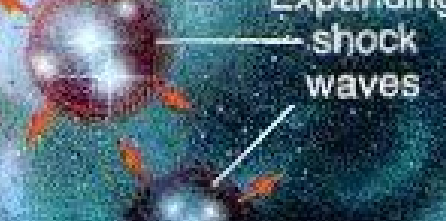

ist-generation stars

Frank Grupp - Spectral synthesis basics

Slide 7 


\section{Element abundances (2)}

- We can hope to find trends with "time" as:

- The timescales for SN are different belonging on type and progenitor mass

- The process goes on recycling material again and again

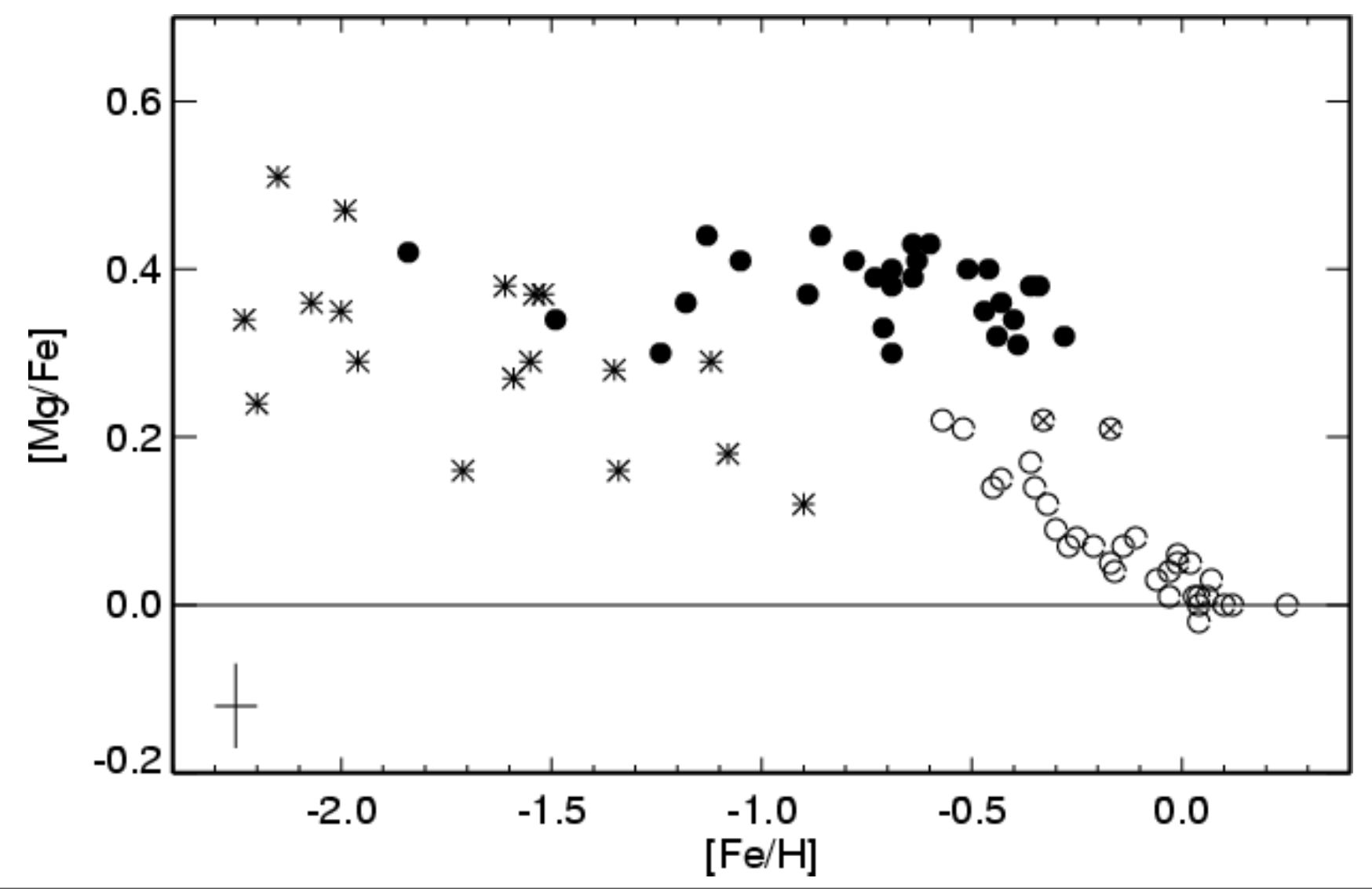




\section{Element abundances (3)}

- How is the knowledge about the past preserved in stars?

- Cool stars evolve really slow

- No mixing of interior and atmosphere for cool stars

- Only diffusion and gravitational settling at work

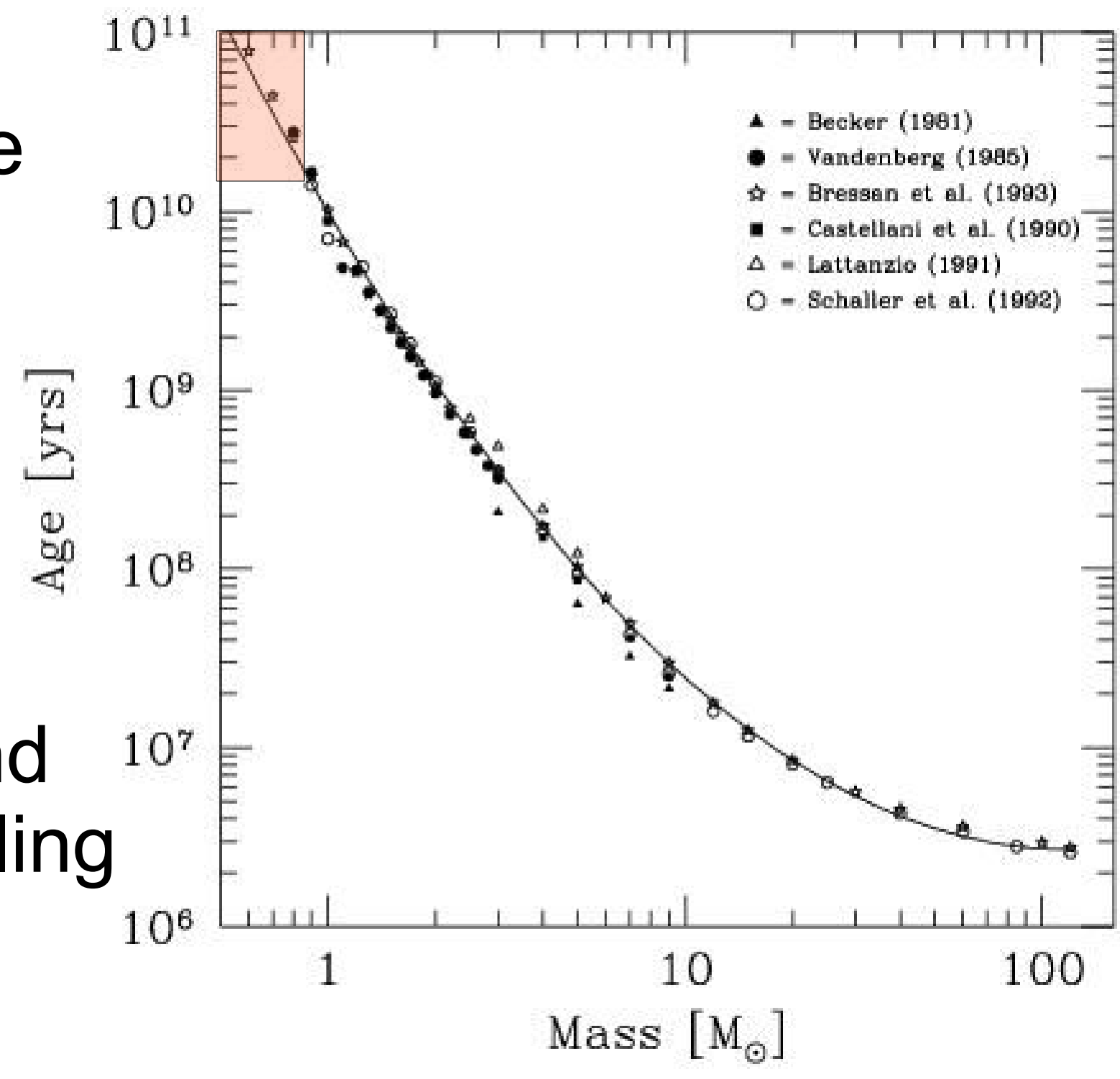




\section{Element abundances (4)}

- So we have interesting questions to answer:

- How did the universe enrich with heavy elements?

- What stellar masses dominated the past?

- How did the first stars "look" like?

- How can we understand quiet and explosive nucleosynthesis?

- Where did the material that we consist of come from? 


\section{Element abundances (5)}

- Definition: Element abundances are usually measured on a logarithmic mass scale, defining the abundance of Hydrogen $\varepsilon(\mathrm{H})=12$.

- On this scale He has an abundance of 11 


\section{Element abundances (6)}

- The solar mixture:

\begin{tabular}{lrlrlrll}
\hline \hline elem & abun & elem & abun & elem & abun & elem & abun \\
\hline $\mathrm{H}$ & 12.00 & $\mathrm{He}$ & 11.00 & $\mathrm{Li}$ & 3.31 & $\mathrm{Be}$ & 1.42 \\
$\mathrm{~B}$ & 2.79 & $\mathrm{C}$ & 8.55 & $\mathrm{~N}$ & 7.97 & $\mathrm{O}$ & 8.87 \\
$\mathrm{~F}$ & 4.48 & $\mathrm{Ne}$ & 8.08 & $\mathrm{Na}$ & 6.32 & $\mathrm{Mg}$ & 7.58 \\
$\mathrm{Al}$ & 6.49 & $\mathrm{Si}$ & 7.56 & $\mathrm{P}$ & 5.53 & $\mathrm{~S}$ & 7.20 \\
$\mathrm{Cl}$ & 5.28 & $\mathrm{Ar}$ & 6.52 & $\mathrm{~K}$ & 5.13 & $\mathrm{Ca}$ & 6.35 \\
$\mathrm{Sc}$ & 3.10 & $\mathrm{Ti}$ & 4.94 & $\mathrm{~V}$ & 4.02 & $\mathrm{Cr}$ & 5.69 \\
$\mathrm{Mn}$ & 5.53 & $\mathrm{Fe}$ & 7.50 & $\mathrm{Co}$ & 4.91 & $\mathrm{Ni}$ & 6.25 \\
$\mathrm{Cu}$ & 4.29 & $\mathrm{Zn}$ & 4.67 & $\mathrm{Ga}$ & 3.13 & $\mathrm{Ge}$ & 3.63 \\
$\mathrm{As}$ & 2.37 & $\mathrm{Se}$ & 3.38 & $\mathrm{Br}$ & 2.63 & $\mathrm{Kr}$ & 3.23 \\
$\mathrm{Rb}$ & 2.41 & $\mathrm{Sr}$ & 2.92 & $\mathrm{Y}$ & 2.23 & $\mathrm{Zr}$ & 2.61 \\
$\mathrm{Nb}$ & 1.40 & $\mathrm{Mo}$ & 1.97 & $\mathrm{Ru}$ & 1.83 & $\mathrm{Rh}$ & 1.10 \\
$\mathrm{Pd}$ & 1.70 & $\mathrm{Ag}$ & 1.24 & $\mathrm{Cd}$ & 1.76 & $\mathrm{In}$ & 0.82 \\
$\mathrm{Sn}$ & 2.14 & $\mathrm{Sb}$ & 1.03 & $\mathrm{Te}$ & 2.24 & $\mathrm{I}$ & 1.51 \\
$\mathrm{Xe}$ & 2.23 & $\mathrm{Cs}$ & 1.13 & $\mathrm{Ba}$ & 2.22 & $\mathrm{La}$ & 1.22 \\
$\mathrm{Ce}$ & 1.63 & $\mathrm{Pr}$ & 0.80 & $\mathrm{Nd}$ & 1.49 & $\mathrm{Sm}$ & 0.98 \\
$\mathrm{Eu}$ & 0.55 & $\mathrm{Gd}$ & 1.09 & $\mathrm{~Tb}$ & 0.35 & $\mathrm{Dy}$ & 1.17 \\
$\mathrm{Ho}$ & 0.51 & $\mathrm{Er}$ & 0.97 & $\mathrm{Tm}$ & 0.15 & $\mathrm{Yb}$ & 0.96 \\
$\mathrm{Lu}$ & 0.13 & $\mathrm{Hf}$ & 0.75 & $\mathrm{Ta}$ & -0.13 & $\mathrm{~W}$ & 0.69 \\
$\mathrm{Re}$ & 0.28 & $\mathrm{Os}$ & 1.39 & $\mathrm{Ir}$ & 1.37 & $\mathrm{Pt}$ & 1.69 \\
$\mathrm{Au}$ & 0.87 & $\mathrm{Hg}$ & 1.17 & $\mathrm{Ti}$ & 0.83 & $\mathrm{~Pb}$ & 2.06 \\
$\mathrm{Bi}$ & 0.71 & $\mathrm{Th}$ & 0.09 & $\mathrm{U}$ & -0.50 & & \\
\hline
\end{tabular}

- "Known" from meteorites and spectroscopy

- Note that C, N and $\mathrm{O}$ abundances have recently been revised

- So this is not a "fixed and verified" table... but work in progress 


\section{Element abundances (7)}

II.II. What do we want to know
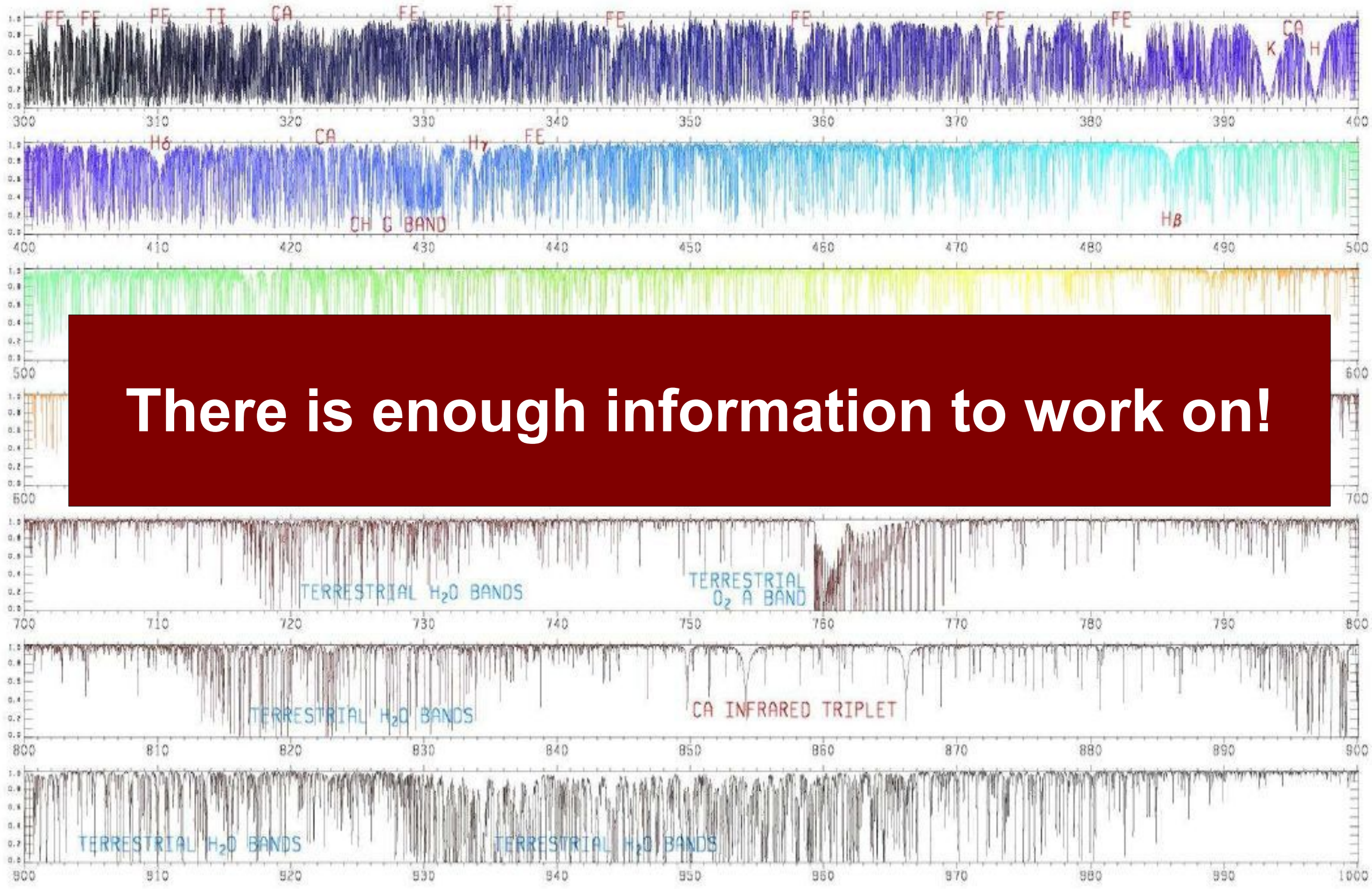

frank@grupp-astro.de

Frank Grupp - Spectral synthesis basics

Slide 13 


\section{Element abundances (8)}
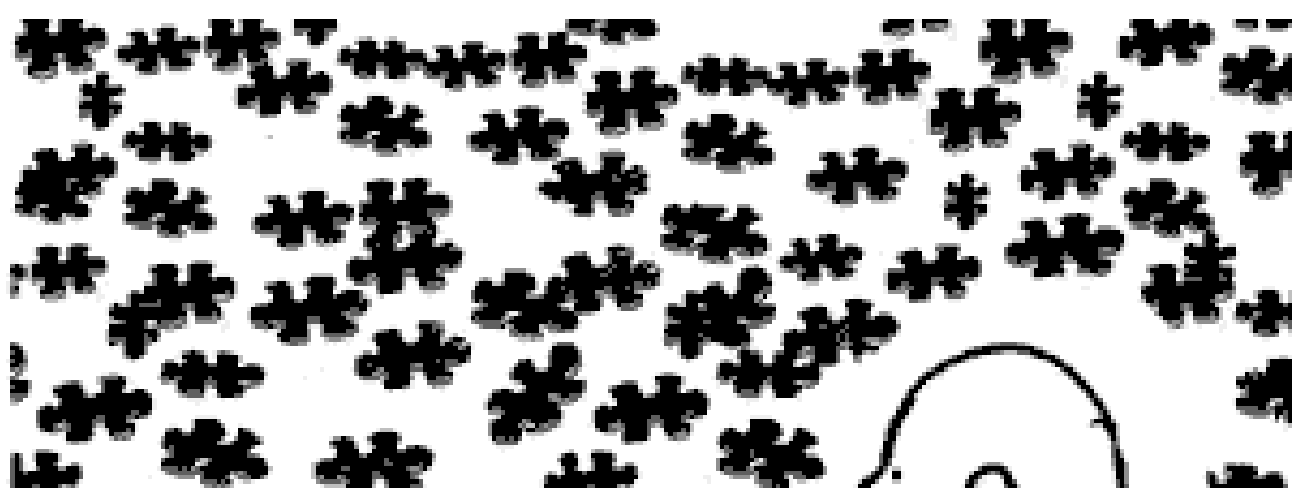

$+2 x+4)$
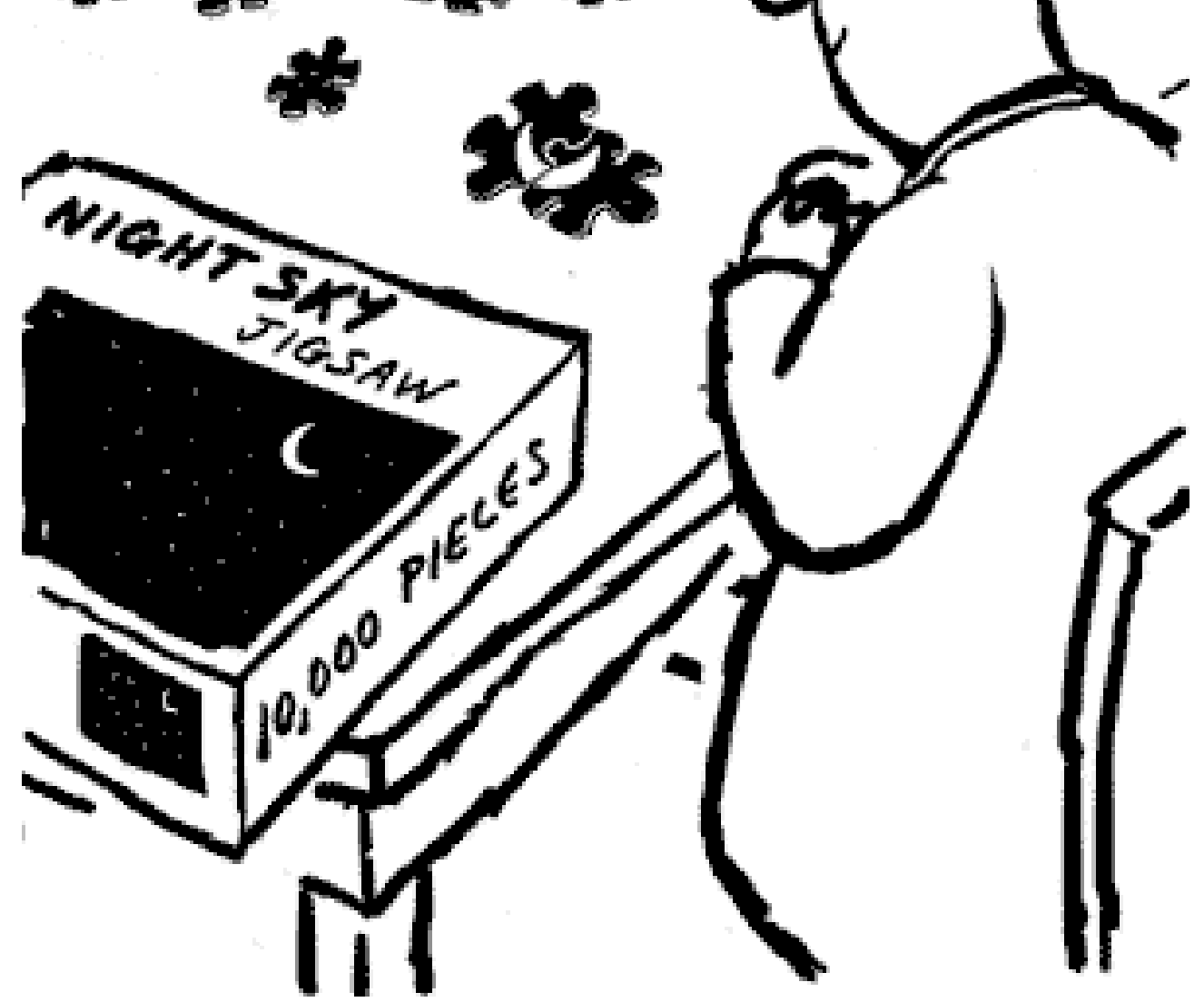


\section{Outline of the lecture}

I. Who I am, what I do.

II.Cool star element abundances

I. Why do we want to know

II.What do we want to know

III.How do we find out

I. The general framework

II.Line formation in model atmospheres

III. The curve of growth

IV.Line fitting

IV.NLTE and why we better use that 


\section{Reality and model (1)}

-What we do in most cases is

- Do measurements

- Calculate a synthetic model representation of the measured quantity

- Compare model and measurement

- Adopt some input values in the model

- Until we reach agreement

- But be aware that there are uncertainties entering from both "sides"

- From observation

- From synthetic observable generation 


\section{Reality and model (2)}

- There were some model assumption needed to reduce our data

- Even the best reduced data will carry your fingerprint and some undetected features that do not come from your desired target of observation 


\section{Reality and model (3)}

- So the reality of your measurement is not an objective one

- Lets keep that in mind all the time working with data

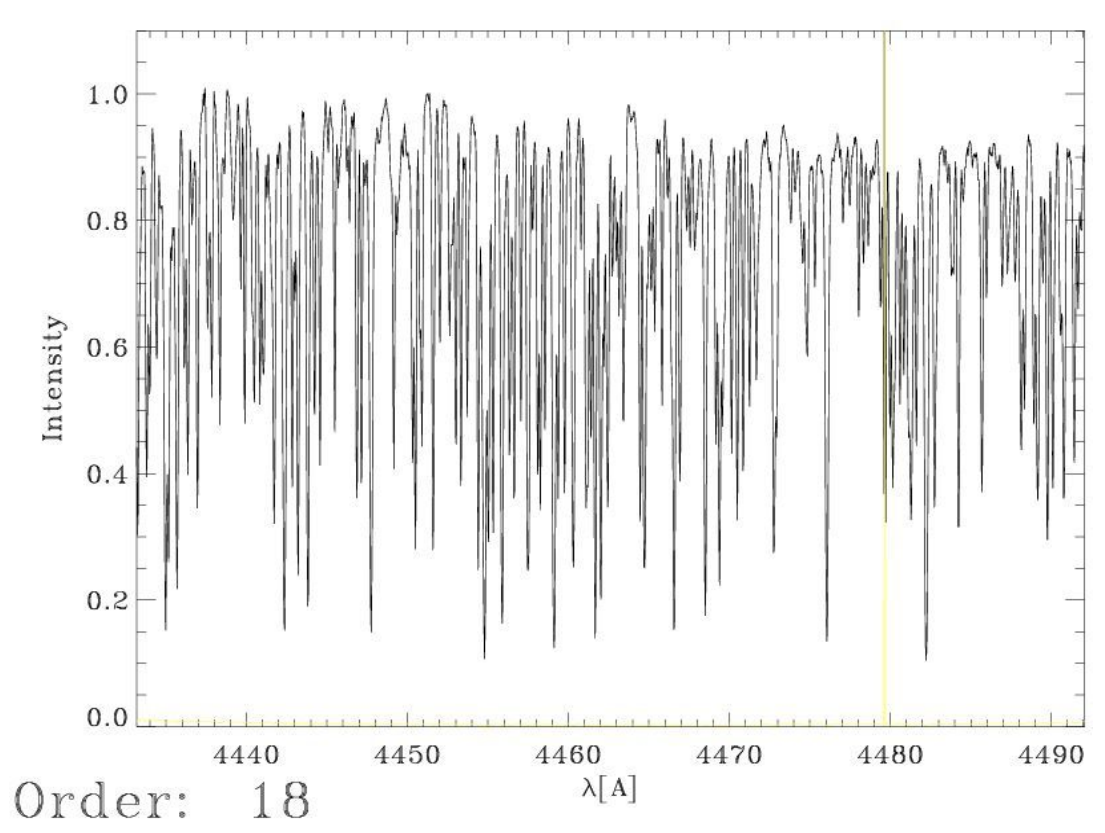




\section{Reality and model (4)}

- On the other hand loads of assumptions, empirical knowledge and decisions what method to use enter the modeling process

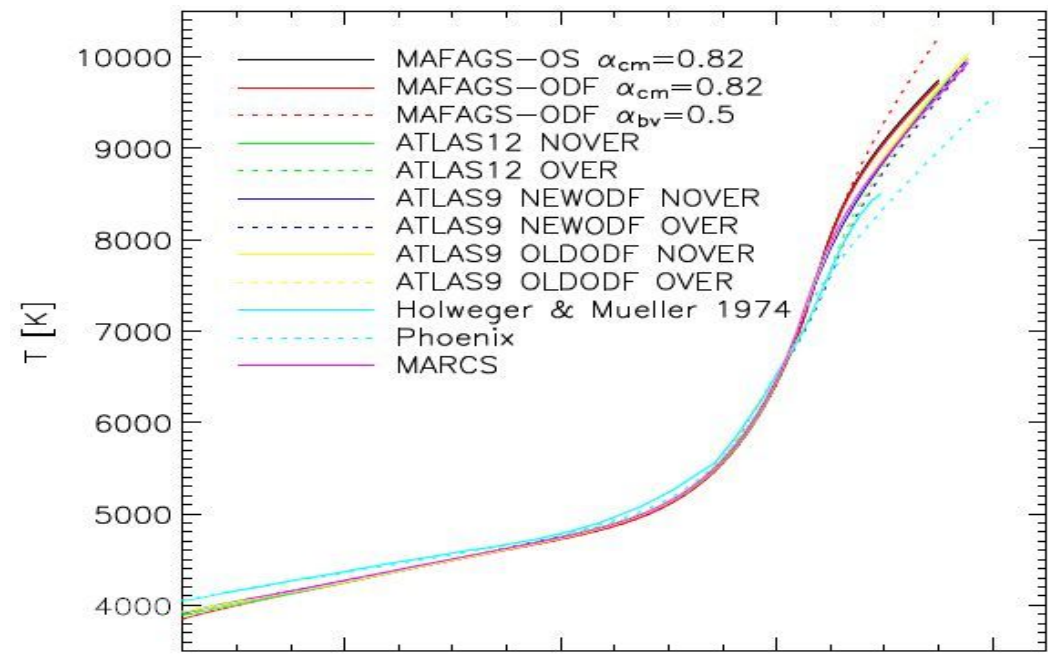

- What sort of model atmospheres do we use

-What sort of convectional treatment

- LTE or Non-LTE

-Where does our atomic data come from

- Are we using measured or theoretical atomic data 


\section{Reality and model (5)}

- In most cases neither observed quantities nor the synthetic representation are absolute in any way

- Both, observed data an model representation contribute to the errors

- Its my personal opinion, that with modern telescopes and spectrographs the latter starts to dominate in many cases

- Although we will not have the appropriate time to do so (today) we should be aware, that a measurement without an appropriate error bar is not worth much 


\section{Synthetic data - a sketch (1)}

III.I. The general framework
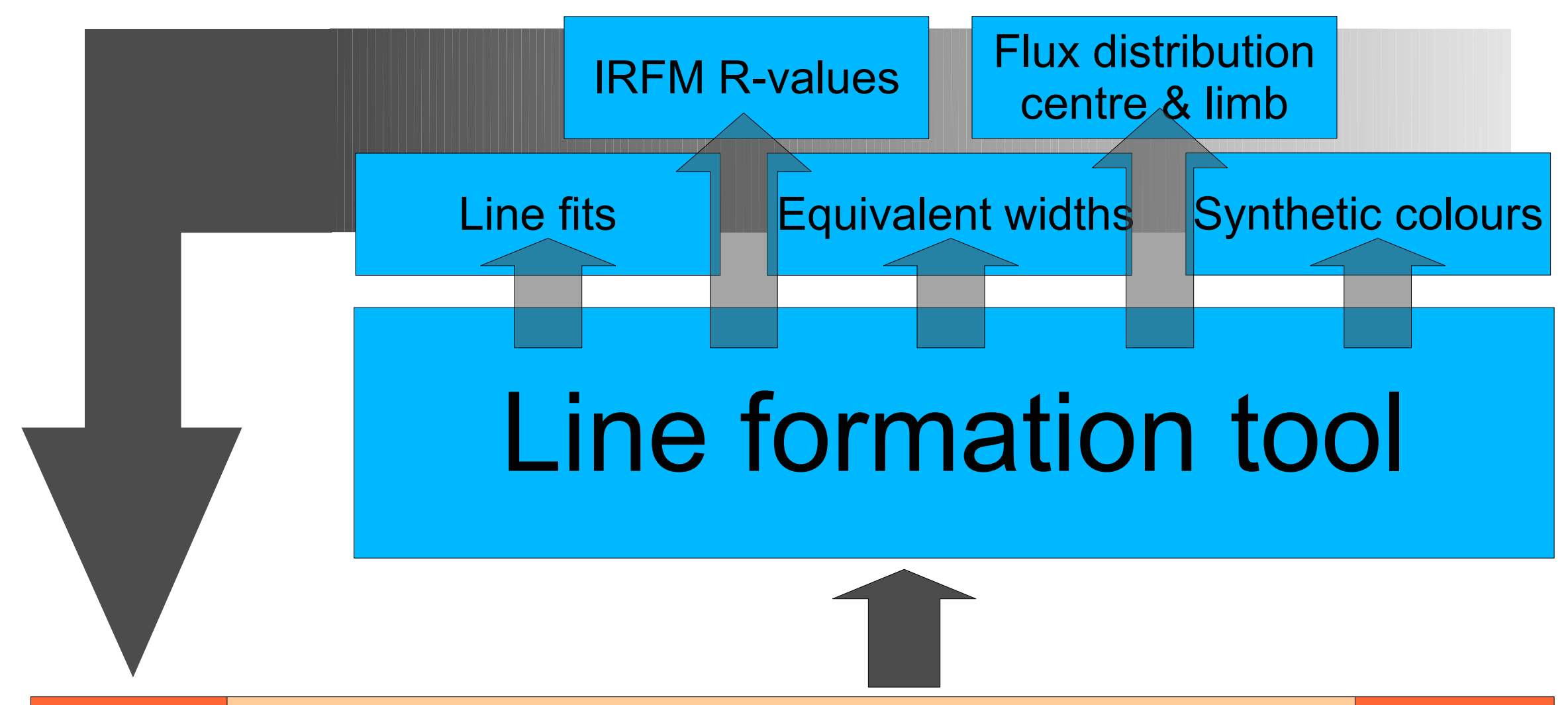

Stellar parameters:

Teff, $\log (g),[M / H],\left[X_{i} / H\right]$, Gmicro

\section{Model atmosphere}




\section{Synthetic data - a sketch (2)}

III.I. The general framework

- Model atmospheres are the backbone of almost all methods of spectroscopy and synthetic photometry

- There are different approaches to model atmospheres

- They lead to different results

- All results will de-

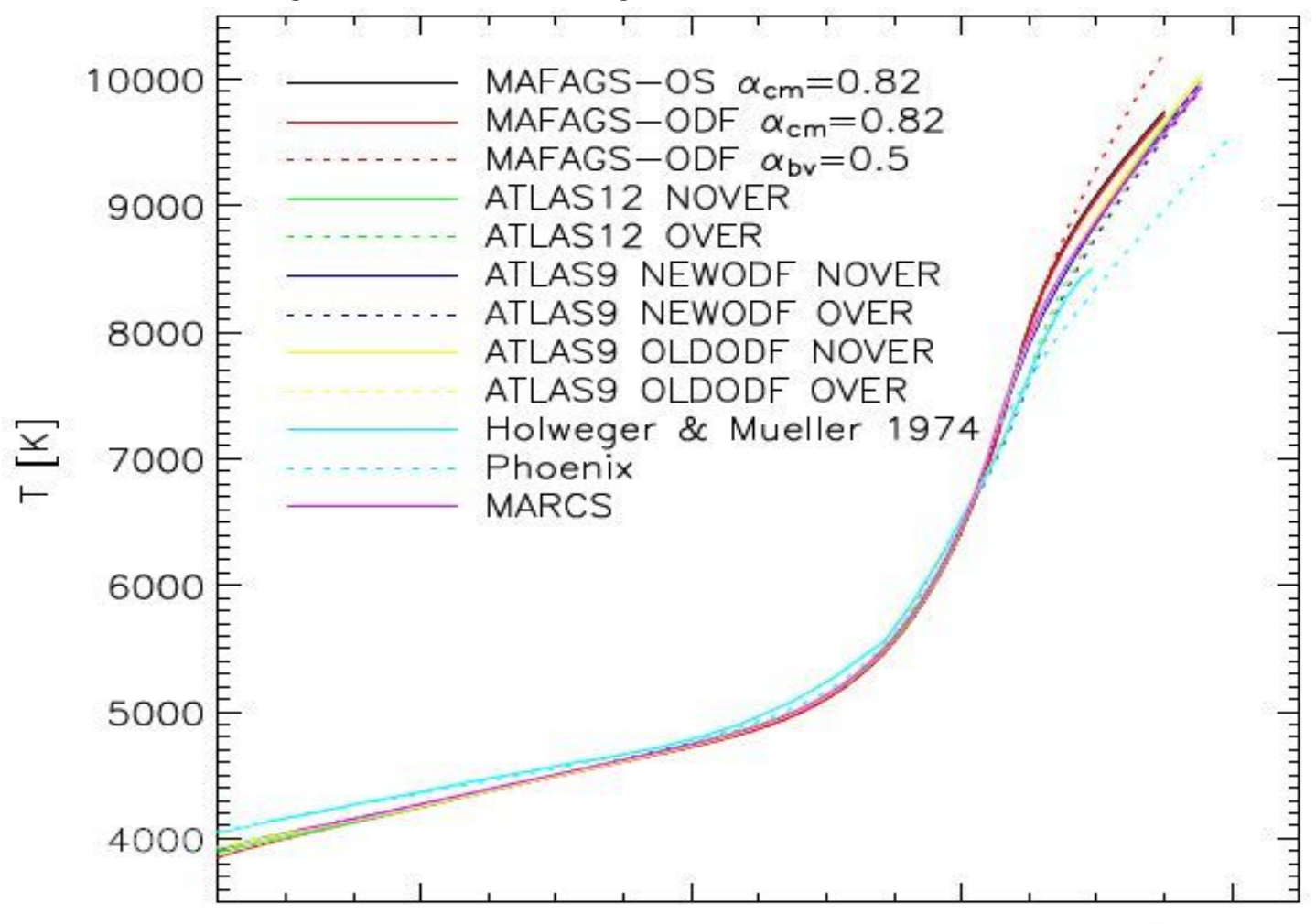
pend on the model atmosphere used

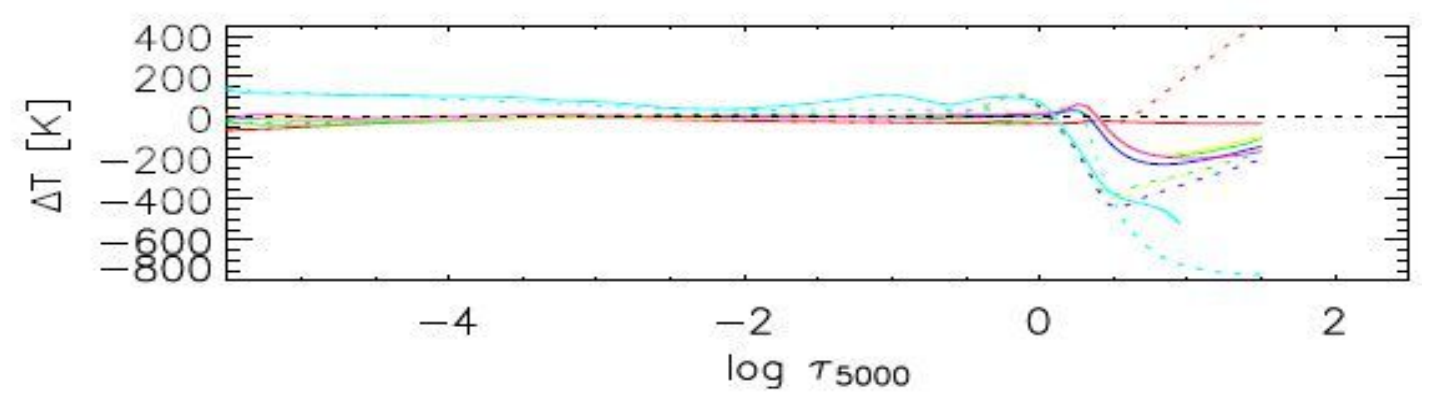




\section{Outline of the lecture}

I. Who I am, what I do.

II.Cool star element abundances

I. Why do we want to know

II.What do we want to know

III.How do we find out

I. The general framework

II.Line formation in model atmospheres

III. The curve of growth

IV.Line fitting

IV.NLTE and why we better use that 


\section{Spectra basics (1)}

III.II. Line formation

\section{-What is it that shapes our spectra?}

- The temperature of the star through blackbody radiation

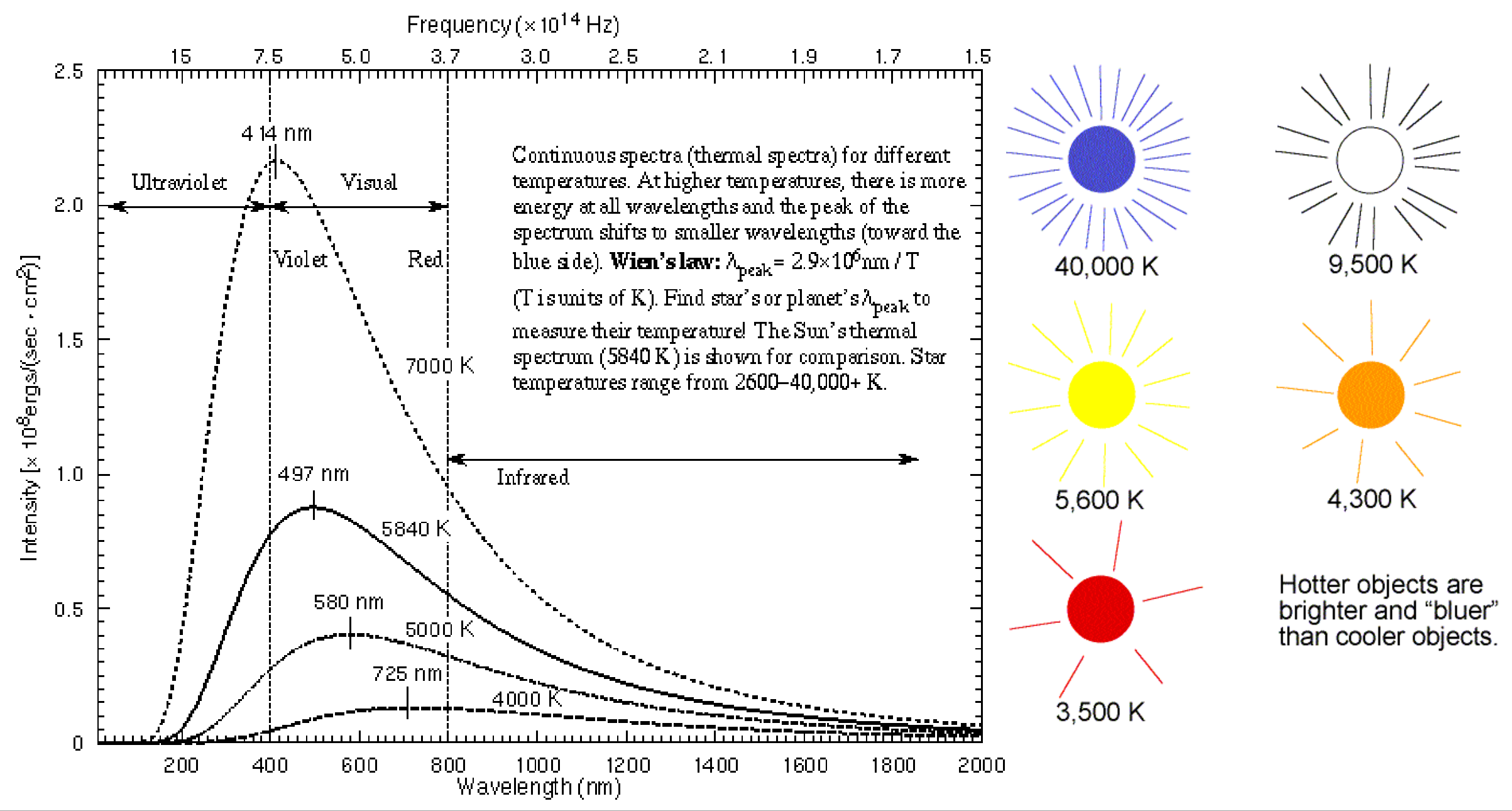

frank@grupp-astro.de

Frank Grupp - Spectral synthesis basics

Slide 24 


\section{Spectra basics (2)}

- But lines appear in our spectra

- In absorption

- In emission

absorption line spectrum

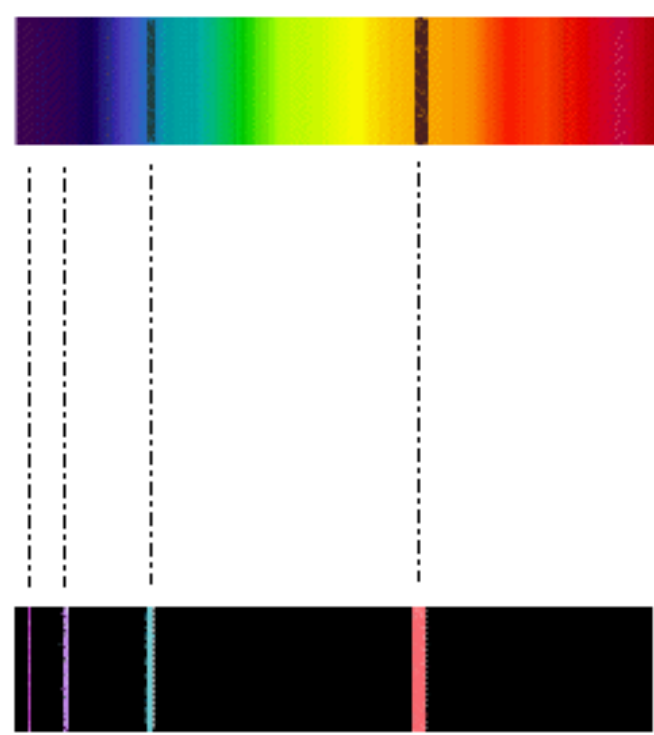

emission line spectrum
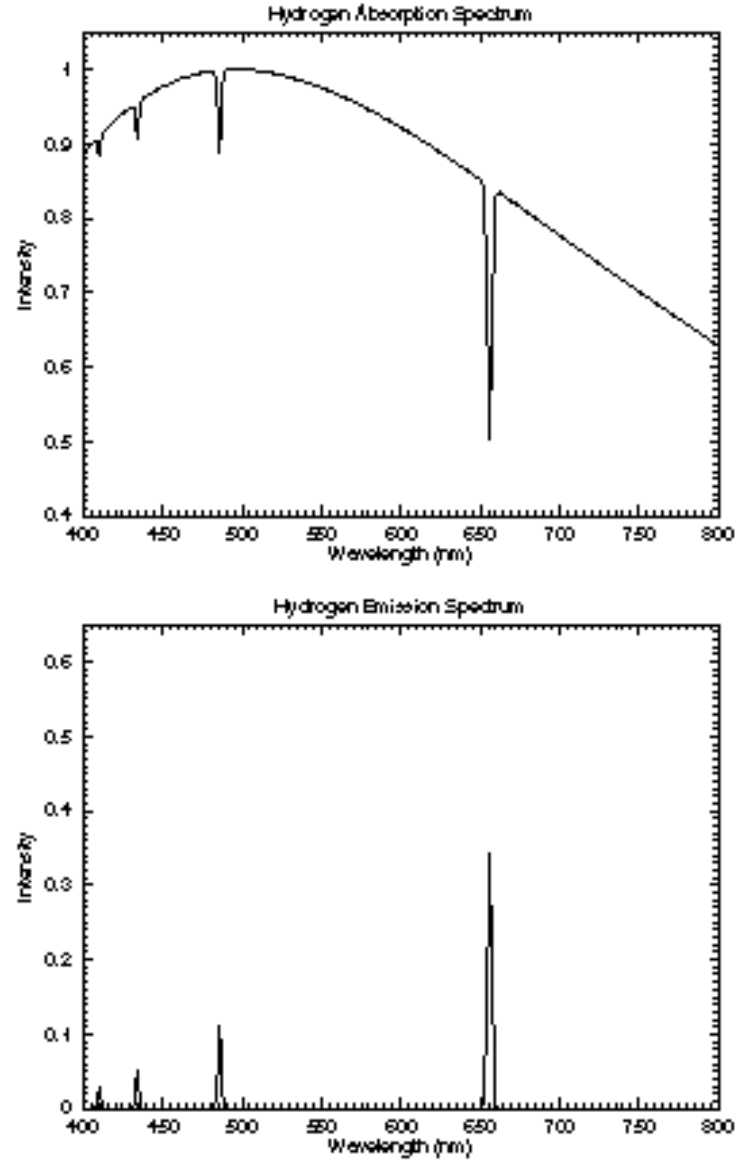

- How can we understand and make use of this effect? $\rightarrow$ How can we do spectroscopy? 


\section{Spectra basics (3)}

- Absorption line spectra

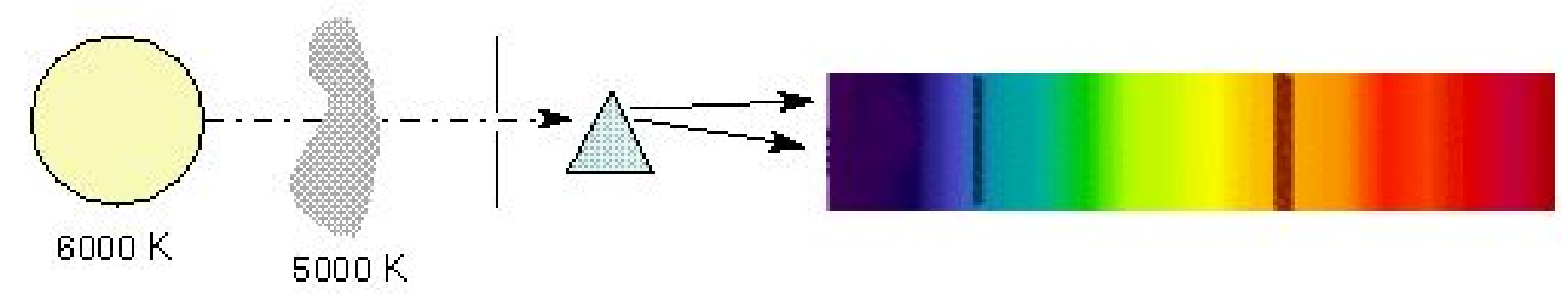

- Are formed if gas with temperatures lower than the background source is located between source and observer

- For stellar atmospheres this means, we find absorption if the temperature of the photosphere has a falling gradient towards the outer layers

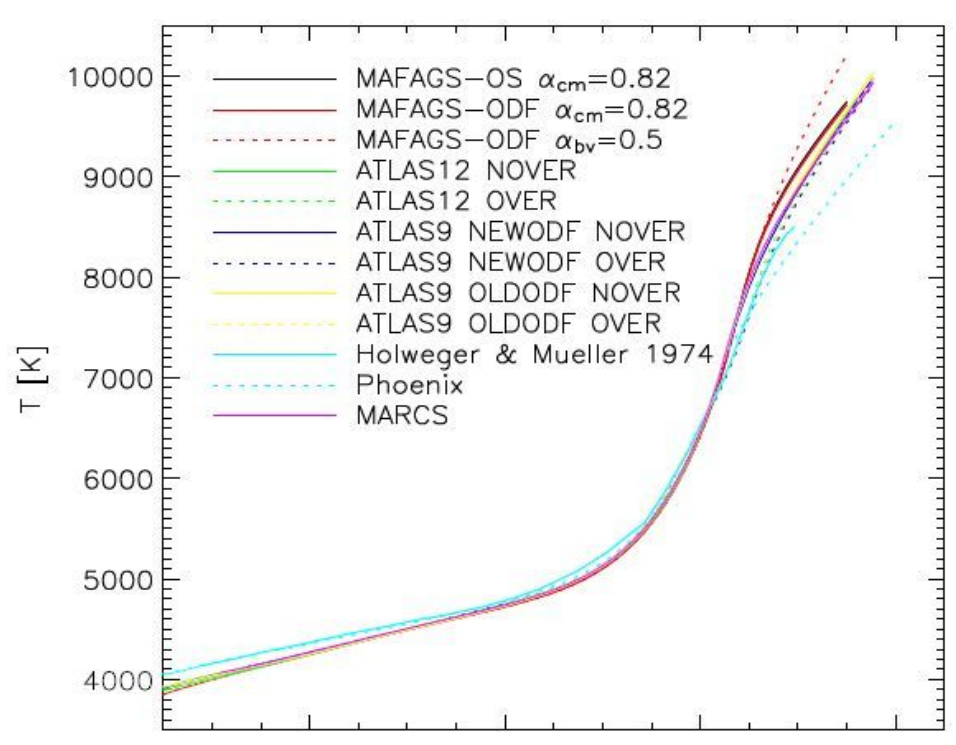




\section{Spectra basics (4)}

- Emission line spectra

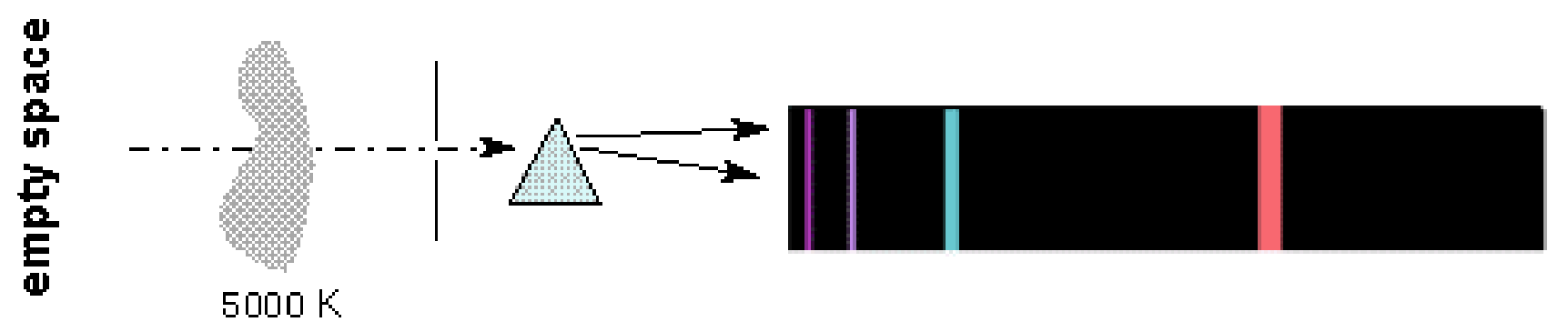

- We will only deal with absorption lines in this lecture!

Emission is of few importance in the stars we look at.

- ror stellar atmospneres tnis means, we find emission if the temperature gradient towards the outside is rising. This is the case in the solar chromosphere and corona . 


\section{Spectra basics (5)}

\section{- A comic type look at absorption}
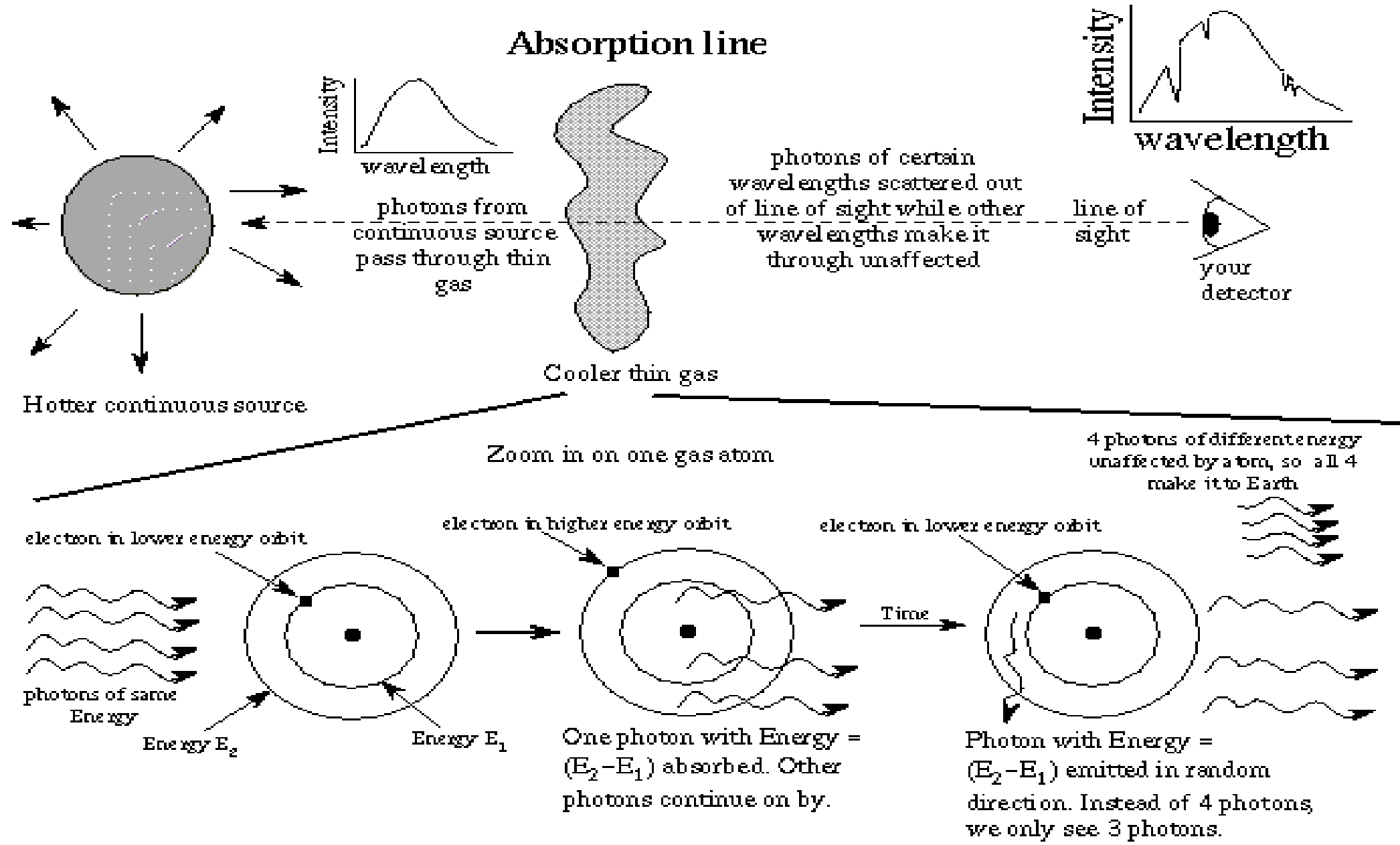


\section{Take a deep breath}

III.II. Line formation

\section{- Now come the details}

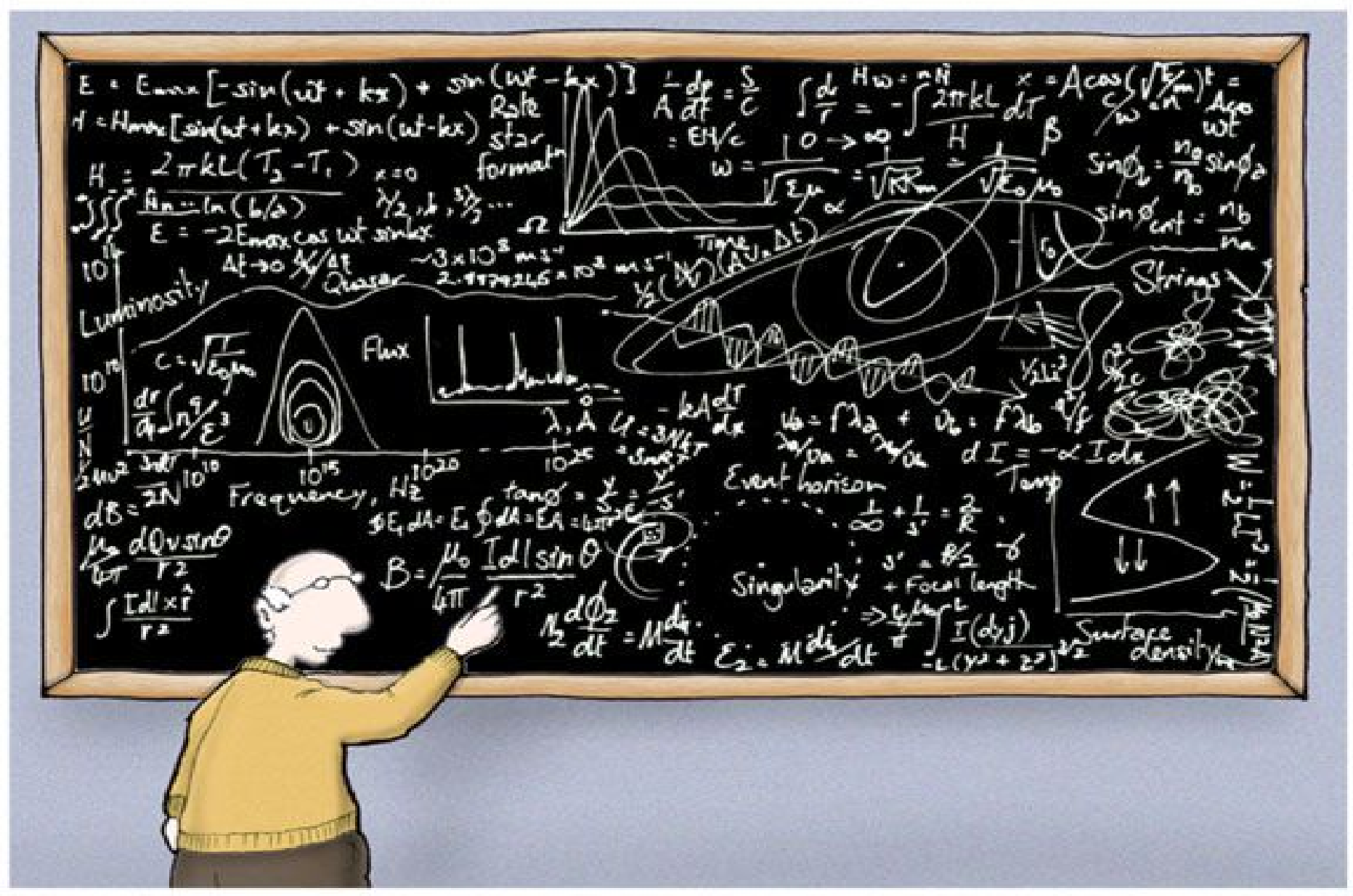

Astrophysics made simple 


\section{Optical depth (1)}

- The concept of optical depth

$$
\begin{gathered}
I / I_{0}=\mathrm{e}^{-\tau} \\
\tau=-\ln \left(I / I_{0}\right)
\end{gathered}
$$

- T is defined as the negative logarithm of the fraction of light scattered or absorbed along the path

- We often give $\log (\tau)=\log \left(-\ln \left(I / I_{0}\right)\right)$ as we have to cover a large range of $T$ values

- At $\log (T)=1 \quad(T=10)$ only a fraction $0.005 \%$ of the initial intensity remains. 


\section{Optical depth (2)}

- Optical depth and absorption/opacity

$$
\begin{aligned}
& d \tau=-K d z \quad d T: \text { Change in opt. Depth } \\
& d \tau=-\kappa \rho d z \\
& d \tau=-\kappa \rho \cos (\Theta) d z
\end{aligned}
$$




\section{Optical depth (3)}

- Calculating $T$ by integration

$$
\tau=\int_{0}^{z} K d z
$$

- So we have shifted the problem

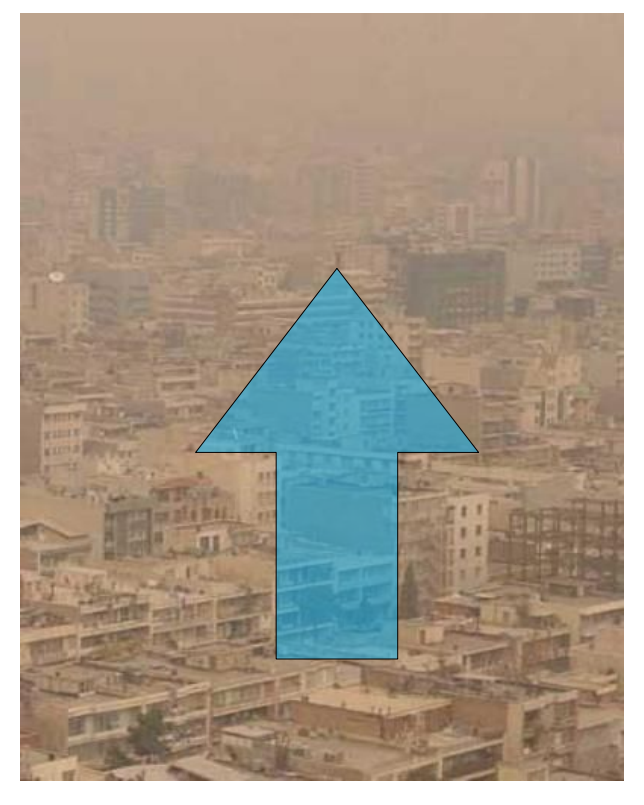

- If we know $T(\lambda)$ we know I $(\lambda)$ (what we want)

- If we know $\mathrm{k}(\lambda)$ we know $\mathrm{T}(\lambda)$

- So we have to calculate $k(\lambda)$ for every $\lambda$

- That is the main task of spectrum synthesis! 


\section{Line absorption (1)}

- About Einstein coefficients

- In radiative equilibrium spontaneous absorption from $L 1$ to $L 2$ is balanced by spontaneous emission from $L 2$ to $L 1$

$$
A_{12}=-A_{21}
$$




\section{Line absorbtion (2)}

- The rate for spontaneous absorption is given by

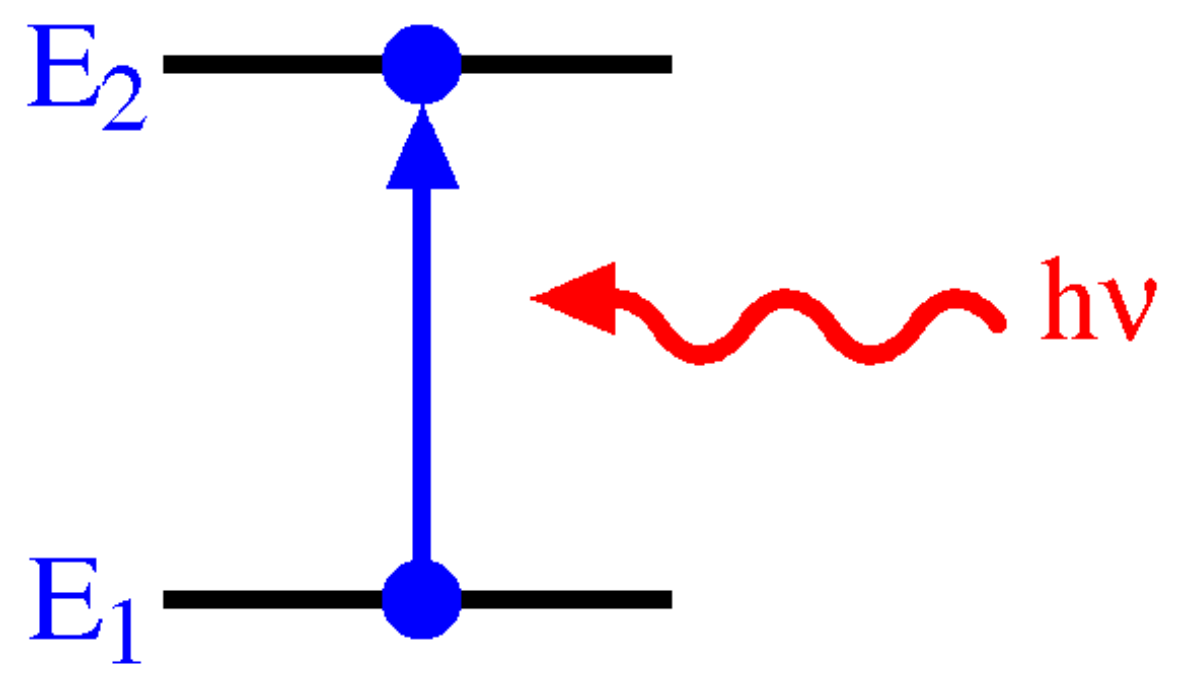

Sp. Abs. Rate $=n_{1} B_{12} b_{v}$

$$
B_{12}=\frac{g_{2}}{g_{1}} \frac{c^{2}}{2 \mathrm{~h} v} A_{21}
$$

ni: Occupancy of state i $b v$ : Planck brightness

$g_{i:}$ Statistical degeneracy

c: Speed of light

$h$ : Plancks constant

$v$ : Frequency 


\section{Line absorbtion (3)}

- The rate for spontaneous emission is given by

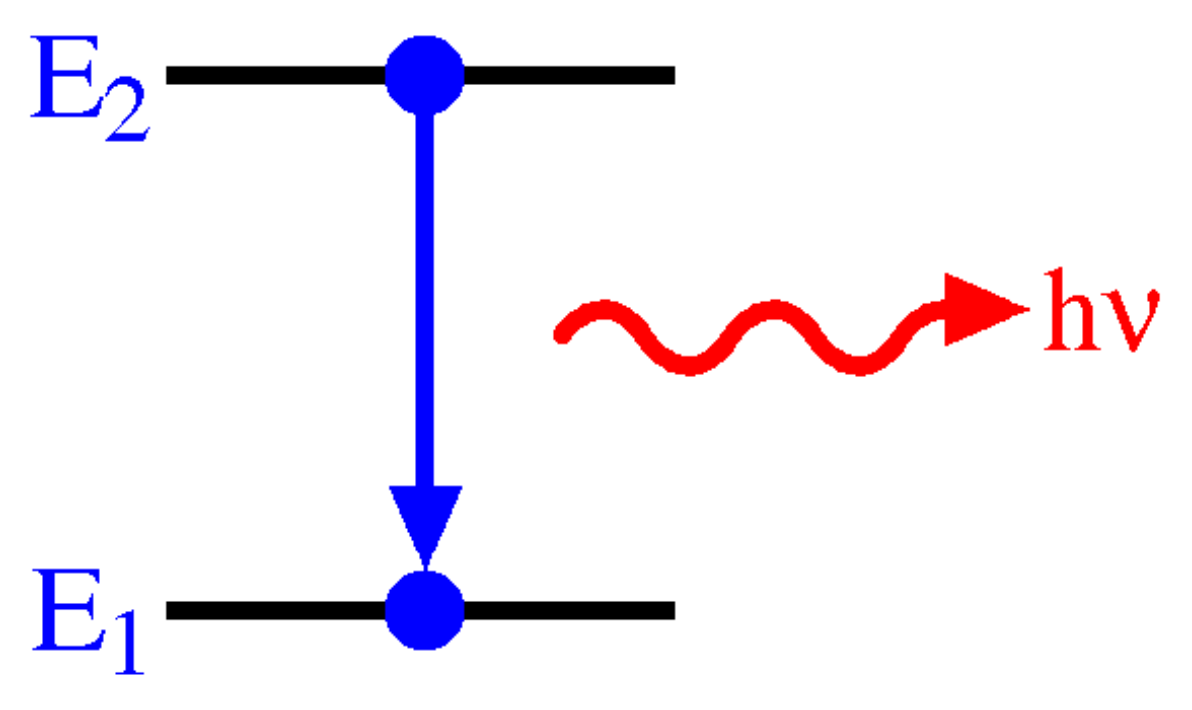

Sp. Emi. Rate $=n_{2} A_{21}$ 


\section{Line absorbtion (4)}

- The rate for stimulated emission is given by

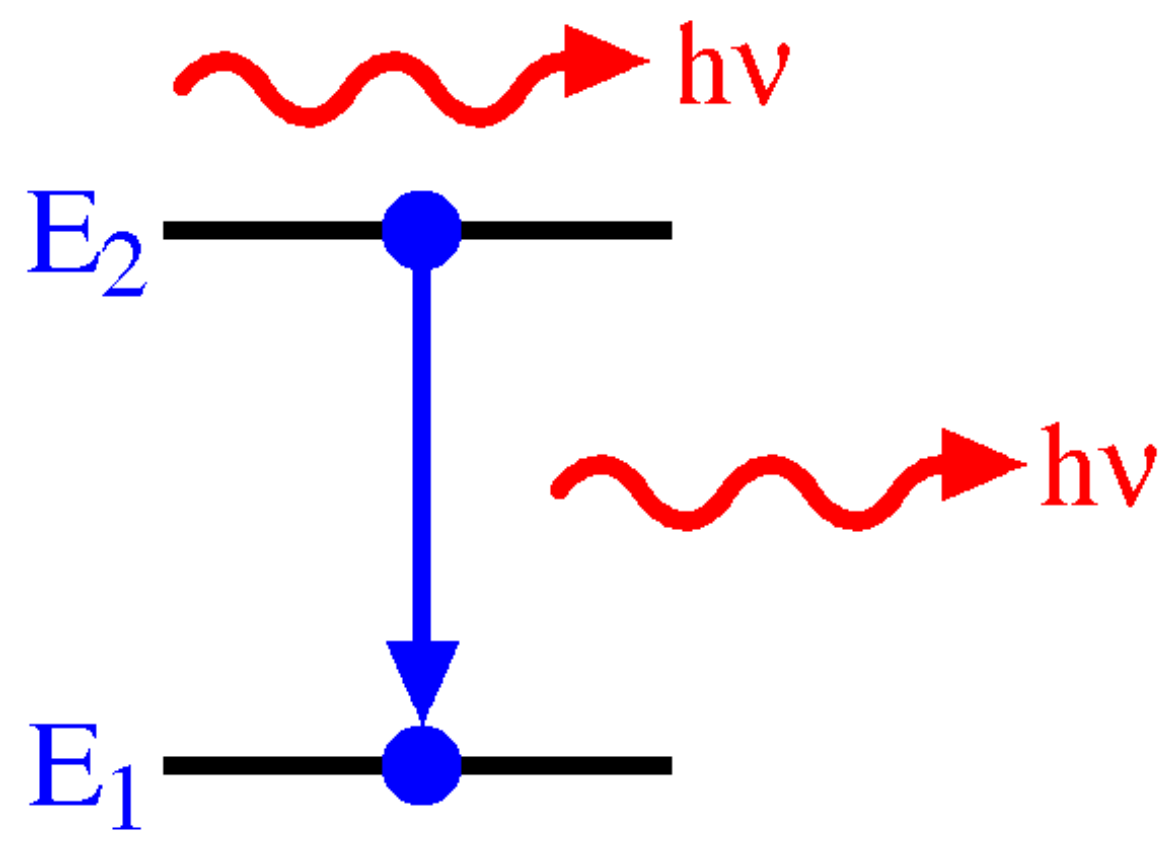

St. Emi. Rate $=n_{2} B_{21} b_{v}$

ni: Occupancy of state i

$b_{v}$ : Planck brightness

$$
B_{21}=\frac{c^{2}}{2 \mathrm{~h} v} A_{21}
$$

$g_{i:}$ Statistical degeneracy

c: Speed of light

$h$ : Plancks constant

$v$ : Frequency 


\section{Line absorbtion (5)}

- Knowing Einstein coefficients we can rewrite the absorbing opacity of a transition

$$
K_{v}=\left(n_{1} B_{12}-n_{2} B_{21}\right) \frac{h v}{4 \pi} \Phi_{v} \quad \Phi_{V: \text { Line profile function }}
$$




\section{Line absorbtion (6)}

- Substituting to $A_{i j}$ coefficients

$$
\begin{gathered}
K_{v}=\left(n_{1} \frac{g_{2}}{g_{1}} \frac{c^{2}}{2 h v^{3}} A_{21}-n_{2} \frac{c^{2}}{2 h v^{3}} A_{12}\right) \frac{h v}{4 \pi} \Phi_{v} \\
K_{v}=\frac{c^{2}}{8 \pi v^{2}} A_{21}\left(\frac{g_{2}}{g_{1}} n_{1}-n_{2}\right) \Phi_{v}
\end{gathered}
$$

- So we know how to calculate the opacity of absorbing atoms form basic Einstein coefficients

- Occupancies of state $1 \& 2 \mathrm{mix}$ in a sum due to stimulated emission 


\section{Line absorbtion (7)}

- Lets rewrite our equations in a more practical form and adopted to the application

- We are interested in line absorption relative to the continuum level

absorption line spectrum

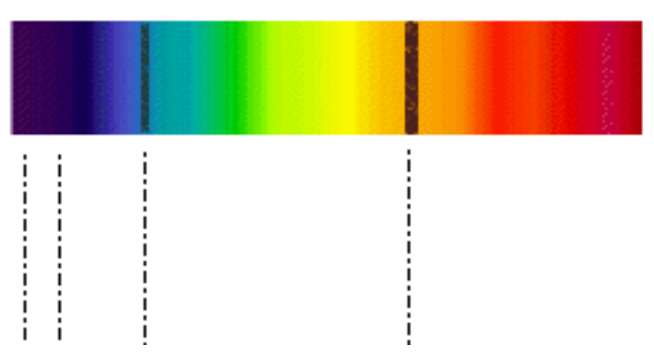

Hutoon Atosption spection

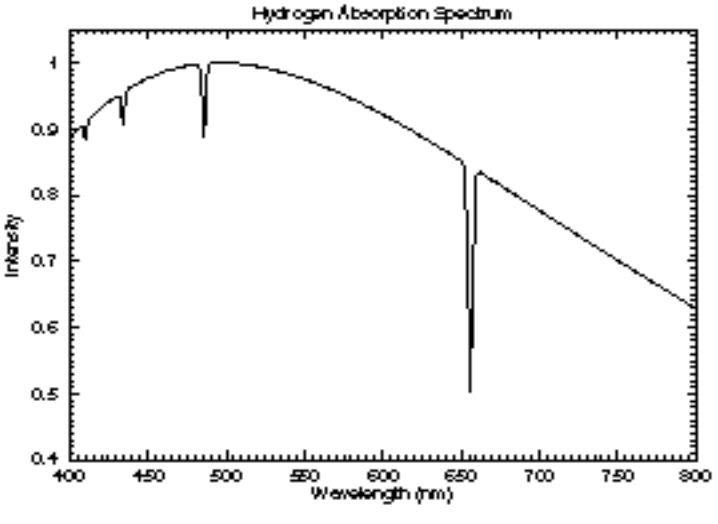

- We define the depth of a line as:

$$
r_{\lambda}(0, \Theta)=\frac{I(0, \Theta)-I_{\lambda}(0, \Theta)}{I(0, \Theta)}
$$

with $\Theta$ the angle of emission for wavelength $\lambda$ 


\section{Line absorbtion (8)}

III.II. Line formation

$$
r_{\lambda}(0, \Theta)=\frac{I(0, \Theta)-I_{\lambda}(0, \Theta)}{I(0, \Theta)}
$$

with the line free continuum:

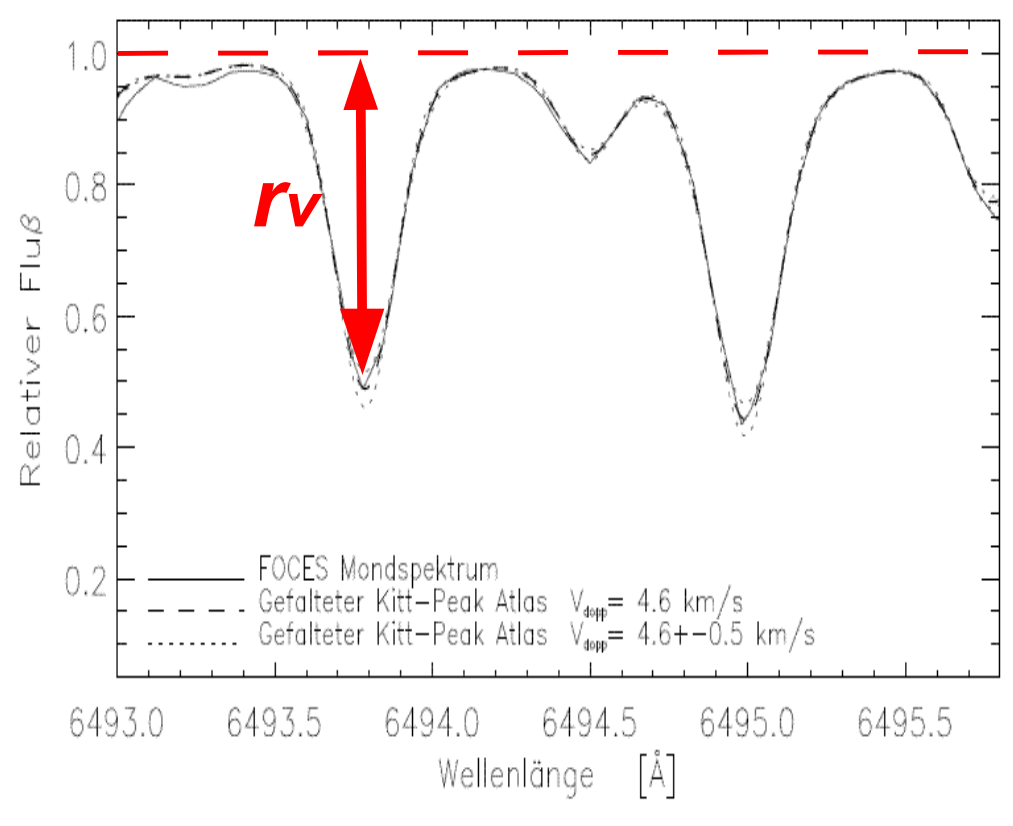

$$
I(0, \Theta)=\int_{0}^{\infty} S(\tau) e^{-\tau / \cos (\Theta)} d \tau / \cos (\Theta)
$$$$
\tau(t)=\int_{0}^{t} \kappa\left(t^{\prime}\right) d t^{\prime}
$$

$T:$ Optical depth

$S(T)$ : Source function (e.g. Planck) $k$ : Coefficient of continuous absorption 


\section{Line absorbtion (9)}

- And the line intensity

$$
\begin{aligned}
& I_{\lambda}(0, \Theta)=\int_{0}^{\infty} S(\tau) e^{-X_{\lambda} / \cos (\Theta)} d X_{\lambda} / \cos (\Theta) \\
& \chi_{\lambda}(0, \Theta)=\int_{0}^{\infty}\left(\kappa_{\lambda}\left(t^{\prime}\right)+\kappa\left(t^{\prime}\right)\right) d t^{\prime}
\end{aligned}
$$

$K \lambda$ : Coefficient of line absorption 


\section{Line absorbtion (10)}

- In model atmospheres of n-layers the integral

$$
I_{\lambda}(0, \Theta)=\int_{0}^{\infty} S(\tau) e^{-X_{\lambda} / \cos (\Theta)} d X_{\lambda} / \cos (\Theta)
$$

changes to a sum

$$
\begin{aligned}
& I_{\lambda}(0, \Theta)=\sum_{\tau_{\min }}^{\tau_{\max }} S(\tau) e^{-X_{\lambda} / \cos (\Theta)} \Delta X_{\lambda} / \cos (\Theta) \\
& X_{\lambda}(0, \Theta)=\sum_{\tau_{\min }}^{\tau_{\max }}\left(\kappa_{\lambda}\left(t^{\prime}\right)+\kappa\left(t^{\prime}\right)\right) \Delta t^{\prime}
\end{aligned}
$$

with finite boundaries 


\section{Line absorbtion (11)}

- Finally we take a rewritten expression of the line absorption coefficient

$$
\kappa_{\lambda}=\frac{1}{4 \pi \epsilon_{0}} \frac{\pi e^{2}}{m c} f N_{n} \Phi(\lambda) E(\lambda, T)
$$

$f:$ "f-value" of the transition $\mathrm{Nn}$ : Occupancy of level $\mathrm{n}$ $E(\lambda, T)$ : Correction function for stimulated emission

$T$ : Temperature

- So we have been hiding a lot of things in the "f-value" of the transition

- We use a function $E(\lambda, T)$ to correct for stimulated emission 


\section{Line absorbtion (12)}

$$
\kappa_{\lambda}=\frac{1}{4 \pi \epsilon_{0}} \frac{\pi e^{2}}{m c} f N_{n} \Phi(\lambda) E(\lambda, T)
$$

- Knowing $f$ and $E$ from atomic physics we still have to learn about

- The occupancy of the state $N_{n}$

- The profile function $\Phi$ that defines the shape of the line 


\section{Continuous absorbers (1)}

$$
X_{v}(0, \Theta)=\int_{0}^{\infty}\left(\kappa_{v}\left(t^{\prime}\right)+\kappa\left(t^{\prime}\right)\right) d t^{\prime}
$$

- We have two major sources of continuous absorption:

- Bound-free interaction / Ionisation

- Free-Free interaction / Photon scatter 


\section{Continuous absorbers (2)}

- Bound-free interaction / Ionisation

- A photon with energy above the ionisation energy of the atom is absorbed

- Extra energy is transferred into kinetic energy of ion and $\mathrm{e}^{-}$

- $\mathrm{K}=0$ for all energies below the ionisation energy

- Above threshold $\mathrm{K} \sim \mathrm{V}^{-3}$

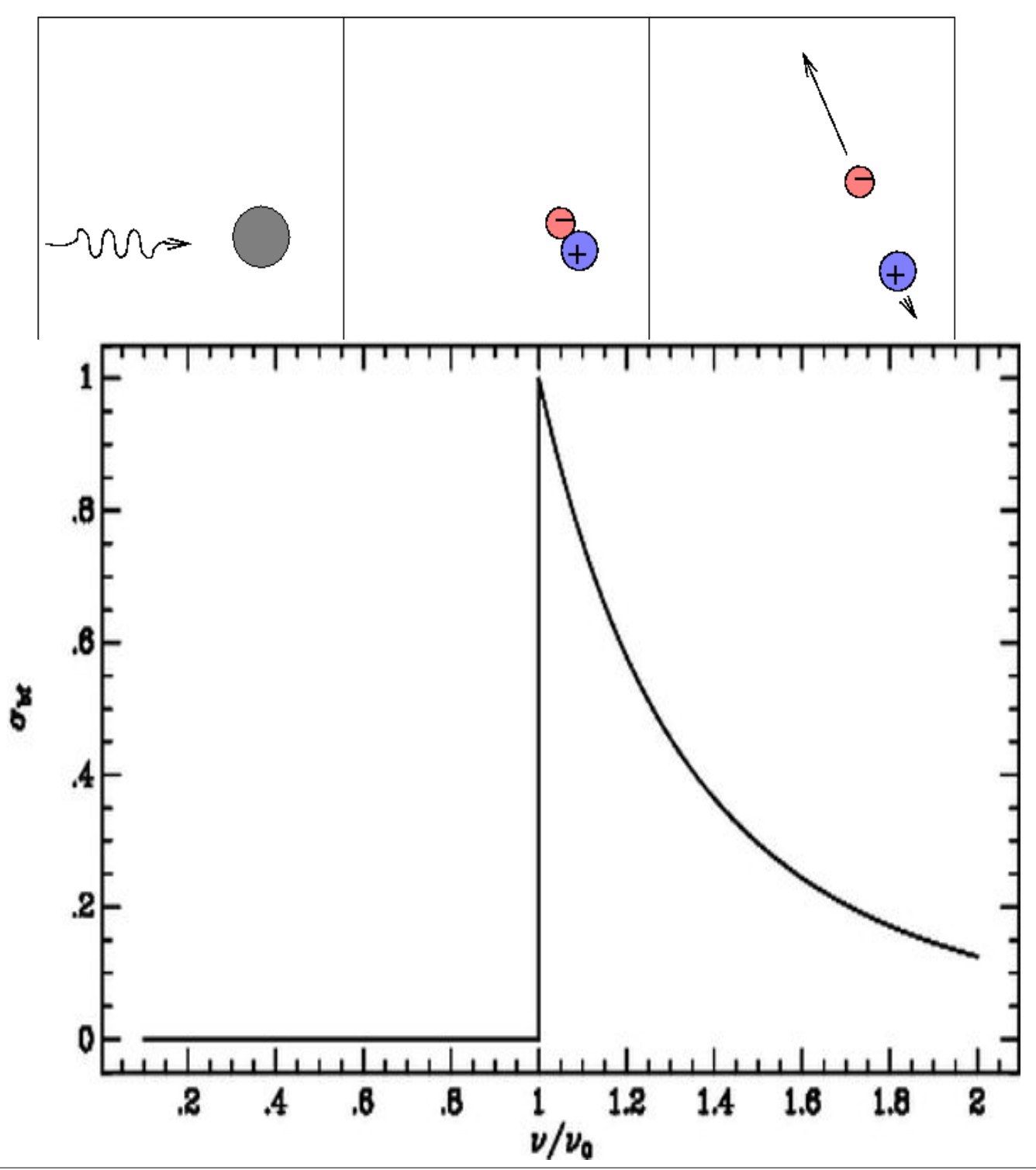




\section{Continuous absorbers (3)}

III.II. Line formation

\section{$B-F$ in the Sun}

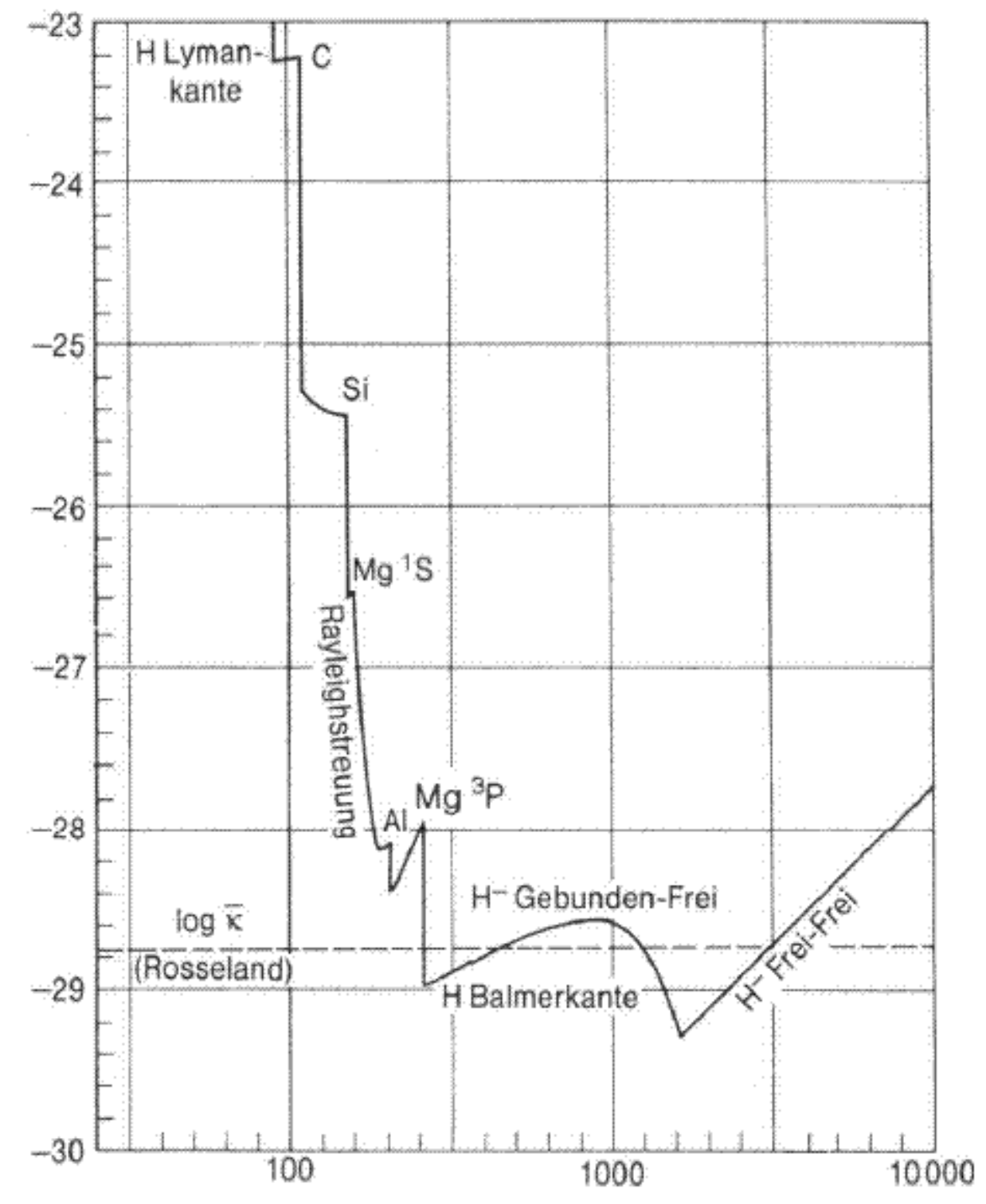

B-F in a hot O-star

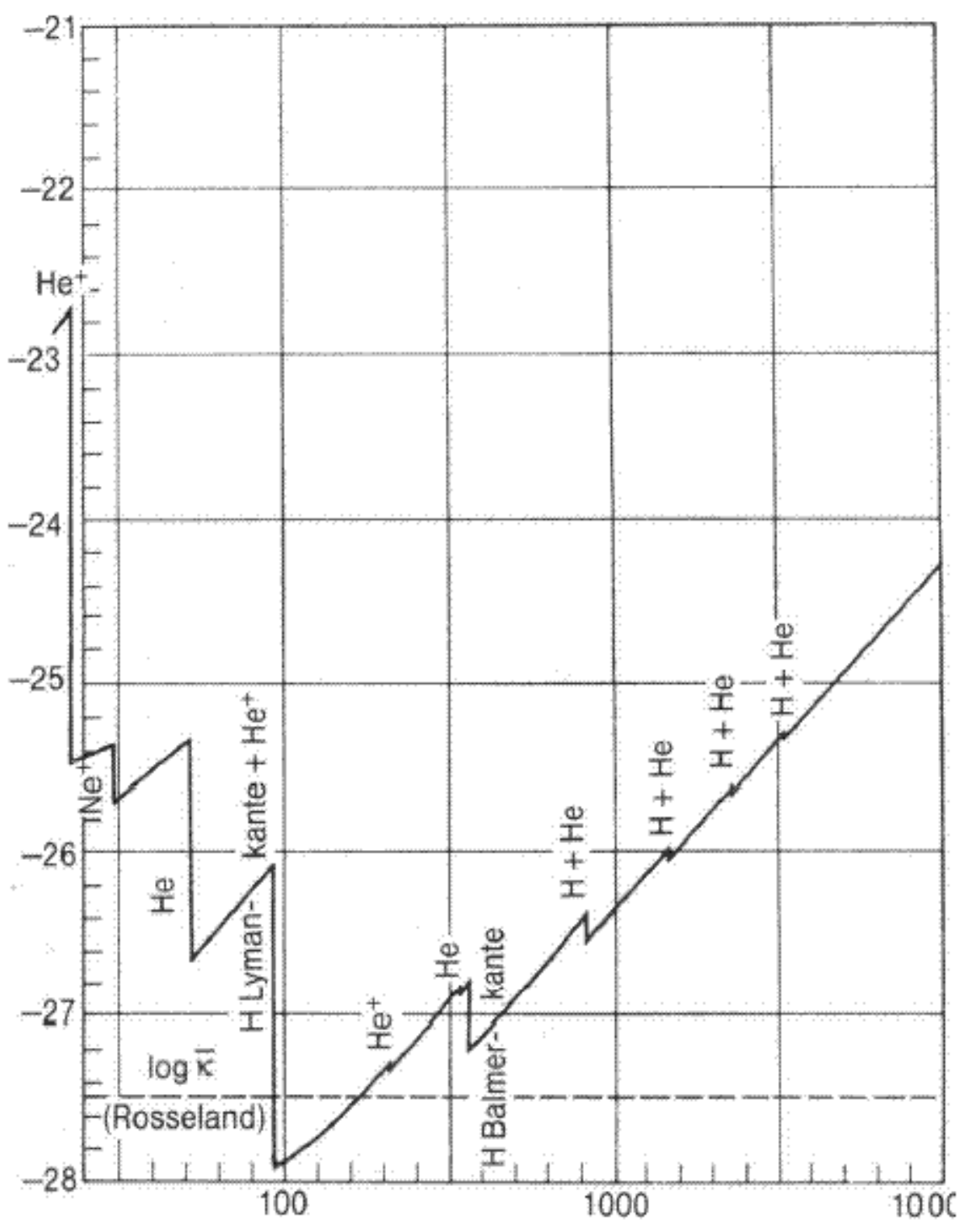




\section{Continuous absorbers (4)}

- Free-Free interaction

- Thompson scatter at charged particles

- Electrons

- $\mathrm{H}$ -

- $\mathrm{H}_{2}-$

- $\mathrm{He}-$

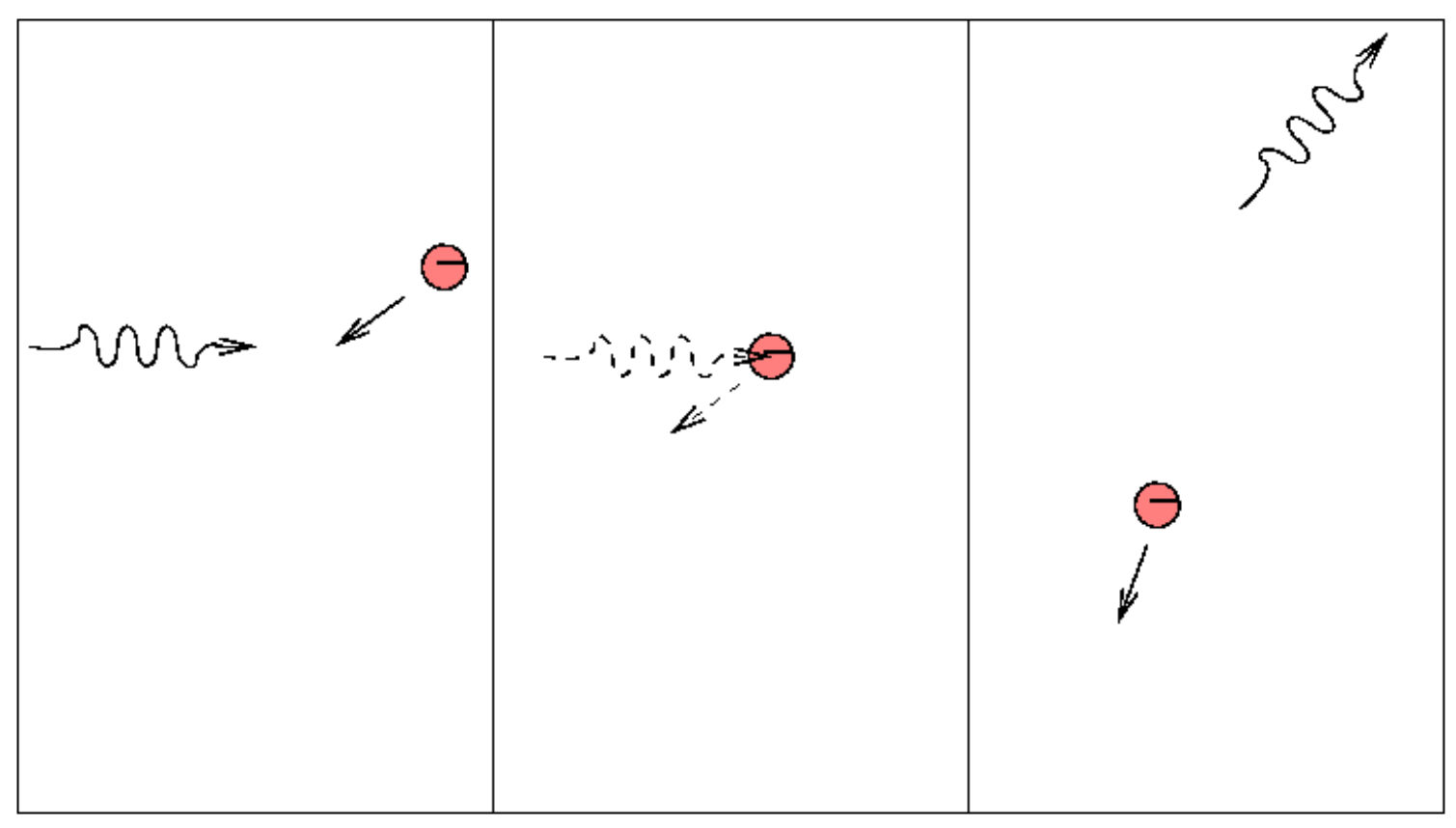




\section{Continuous absorbers (5)}

III.II. Line formation

- Rayleigh scatter at neutral species

- $\mathrm{H}$

- $\mathrm{He}$

- $\mathrm{H} 2$

$-\sigma \sim \lambda^{-4}$

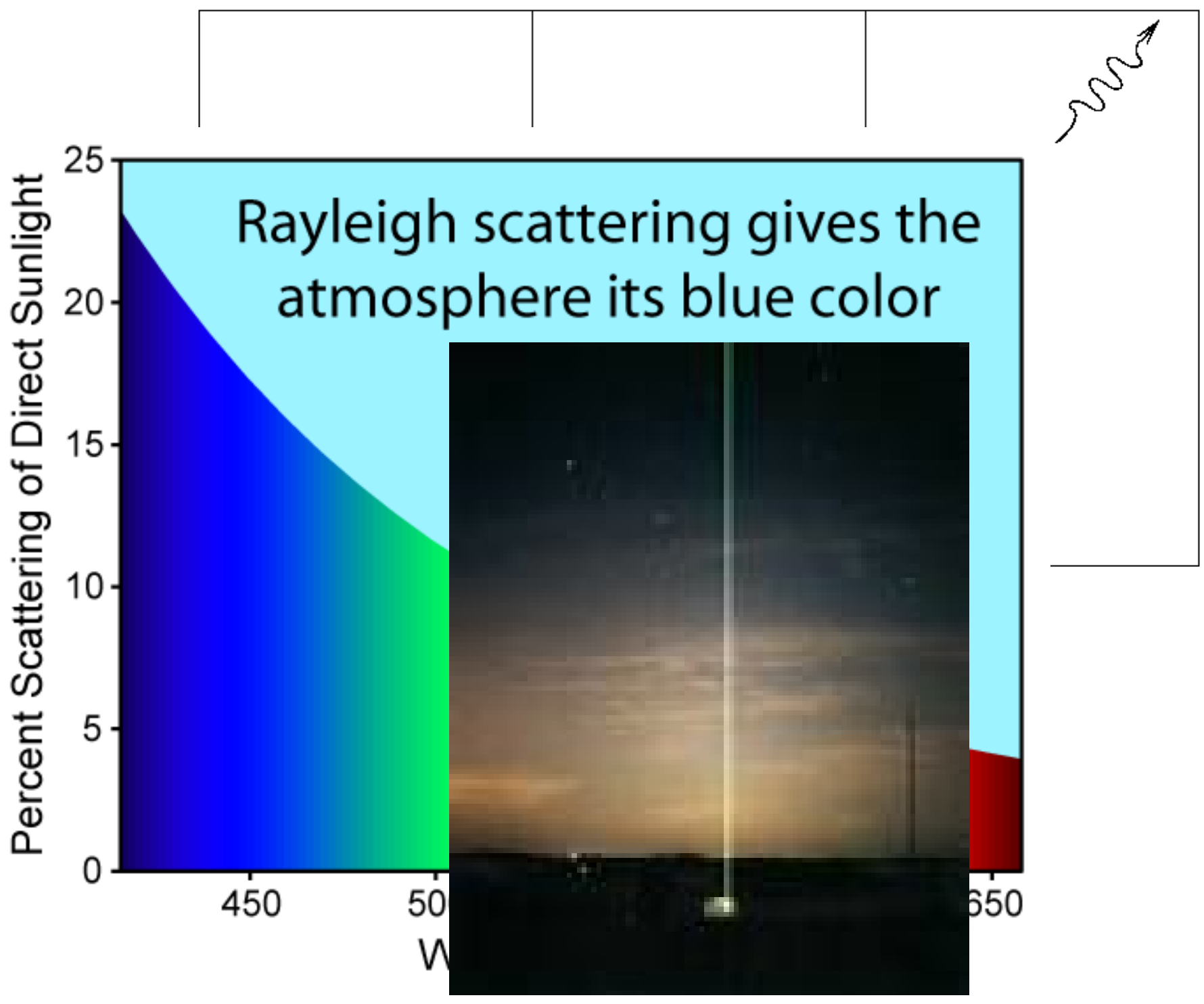




\section{Occupancy (1)}

- In local thermal equilibrium the number of atoms in ionization stage $\mathrm{N}$ and on exaltation level $\mathrm{n}$ is given by the Saha- and Boltzmann equation

- For the occupancy of the exalted level $n$, compared to the ground state 0 the Boltzmann distribution of thermal exaltation leads to:

$$
\frac{N_{0, n}}{N_{0,0}}=\frac{g_{0, n}}{g_{0,0}} e^{-\frac{E_{0, n}-E_{0,0}}{k T}} \quad \begin{aligned}
& g: \text { Statistic weight (multiplicity) } \\
& E: \text { Energy of level } \\
& k: \text { Boltzmann constant } \\
& T: \text { Temperature }
\end{aligned}
$$




\section{Occupancy (2)}

\section{- A numerical example:}

- Na resonance lines:

- $\lambda \approx 5890 \AA \rightarrow E=h c / \lambda=3.37 \mathrm{E}-19 \mathrm{~J}=2.10 \mathrm{eV}$

- $\mathrm{g} 1 / \mathrm{g} 2=6 / 2$

$$
\frac{N_{0, n}}{N_{0,0}}=\frac{g_{0, n}}{g_{0,0}} e^{-\frac{E_{0, n}-E_{0,0}}{k T}}
$$

- $\mathrm{N} 1 / \mathrm{N} 0 \approx 0.05$ 


\section{Occupancy (3)}

- If we include ionization we have to consider a third "species" in addition to ground state and exalted state

$\rightarrow$ the electron that becomes free through ionization

- Lets start with the un-ionized ground-state

$$
\frac{N_{1, n}}{N_{0,0}} N_{e}=\frac{g_{1, n}}{g_{0,0}} g_{e} e^{-\frac{E_{0, n}-E_{0,0}}{k T}}
$$




\section{Occupancy (4)}

- The statistical weight of the electron can be calculated from its extension in phase

$$
g_{e}=2 \frac{\left(2 \pi m_{e} k T\right)^{3 / 2}}{h^{3} N_{e}}
$$

$$
\frac{N_{1, n}}{N_{0,0}} N_{e}=\frac{g_{1, n}}{g_{0,0}} g_{e} e^{-\frac{E_{0, n}-E_{0,0}}{k T}}
$$

- This directly leads to the Saha equation

$$
\frac{N_{1,0}}{N_{0,0}}=\frac{g_{1,0}}{g_{0,0}} 2 \frac{\left(2 \pi m_{e} k T\right)^{3 / 2}}{h^{3} N_{e}} e^{-\frac{x_{0}}{k T}}
$$

$x_{0}$ : lonisation energy 


\section{Occupancy (5)}

- An example

- Fe in the Sun

$$
\frac{N_{1,0}}{N_{0,0}}=\frac{g_{1,0}}{g_{0,0}} 2 \frac{\left(2 \pi m_{e} k T\right)^{3 / 2}}{h^{3} N_{e}} e^{-\frac{x_{0}}{k T}}
$$

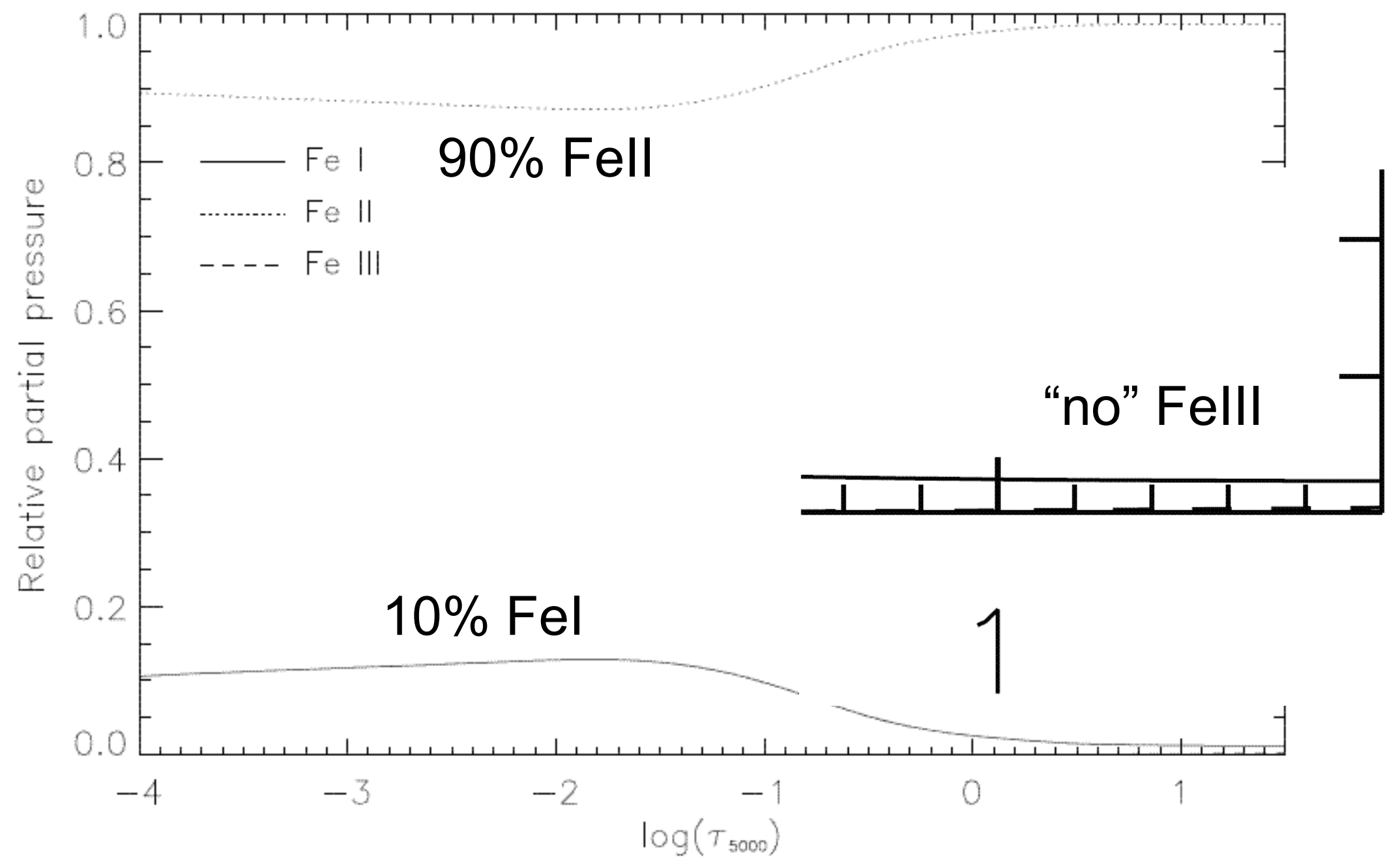




\section{The shape and width of lines (1)}

- After what we have learned about the physics of absorption we still have to determine the shape of the profile function $\Phi(v)$

$$
\kappa_{v}=\frac{1}{4 \pi \epsilon_{0}} \frac{\pi e^{2}}{m c} f N_{n} \Phi(v) E(\nu, T)
$$

- There are several processes contributing to the broadening and shaping of spectral lines 
- The natural line-width / Radiation damping

- Heissenbergs uncertainty principle tells that

$$
\hbar \leq \Delta E \Delta t
$$

- As the lifetime of each level is described by the Einstein coefficients there is a resulting uncertainty in the level Energy

$$
\begin{gathered}
\gamma_{\text {rad }}(n, m)=\sum_{l<n} A_{\mathrm{ln}}+\sum_{l<m} A_{l m} \\
\gamma_{\text {rad }}(n, m): \text { Radiation damping constant } \\
\text { of the n-m transition }
\end{gathered}
$$




\section{The shape and width of lines (3)}

- The shape of the resulting profile is the so called "dispersion profile"

$$
\begin{gathered}
\Phi_{\text {rad }}(\Delta \lambda)=\frac{1}{\pi}\left(\frac{a}{\Delta \lambda^{2}+a^{2}}\right) \\
a=\frac{\lambda^{2}}{4 \pi c} \gamma_{\text {rad }}
\end{gathered}
$$

- As Aij coefficients are of the order of 1e7..1e9 1/s the natural width of a line around $4000 \AA$ is typically $1 \mathrm{e}-$ 5..1e-4A

- Radiation damping plays only a minor role 


\section{The shape and wid
- Collisional broadening}

- Collisions with neutral $\mathrm{H}$ and $\mathrm{He}$ are very likely interactions in cool star atmospheres

- Let us assume a van der Waals potential of the shape:

$$
V_{6}=-\frac{C_{6}}{r^{6}} \quad c_{6}: \mathrm{vdW} \text { damping constant }
$$

- The resulting shift in the energy of level $\mathrm{i}$ will be

$$
\Delta E_{i}=\hbar \frac{C_{6}^{i}}{r^{6}}
$$




\section{The shape and width of lines (5)}

- Unsöld (1955) was able to show that

$$
\begin{gathered}
\gamma_{6}^{i j}=8.08 C_{6}^{0.4} \hat{v}^{0.6} N_{\text {coll }} \\
C_{6}=\left|C_{6}^{i}-C_{6}^{j}\right| \quad \hat{v}=\sqrt{\frac{8}{\pi}\left(\frac{1}{m}+\frac{1}{m_{\text {coll }}} k T\right)}
\end{gathered}
$$

$m$ : Mass of damped species $m_{\text {coll }}$ : Mass of collisional partner $N_{\text {coll }}$ : Densiy of collisional partners

- $\mathrm{C}_{6}$ damping is a major source of line broadening 


\section{The shape and wi
- Doppler broadening}

- Thermal Doppler broadening

- The atoms in the gas of the stellar atmosphere show velocities according to the Maxwell distribution

$$
W(v)=\sqrt{\frac{\mu}{2 \pi k T}} e^{-\frac{\mu v^{2}}{2 \mathrm{RT}}}
$$

$\mu$ : Particle mass

$v$ : Particle speed

- Using the Doppler shift formula

$$
\frac{\Delta \lambda}{\lambda}=\frac{v}{c}
$$

c: Speed of light 
And the definition of Doppler width

$$
I\left(\Delta \lambda_{D}\right)=I_{0} e^{-1}
$$

this leads to the profile

$$
I(\Delta \lambda)=\frac{1}{\sqrt{\pi} \Delta \lambda_{D}} e^{-\frac{\Delta \lambda}{\Delta \lambda_{D}}}
$$

with

$$
\Delta \lambda_{D}=\frac{\lambda}{c} \sqrt{\frac{2 \mathrm{RT}}{\mu}}
$$

Thermal doppler broadening effects the solar Balmer lines (lightest species) by $\Delta \lambda D \approx 0.16 \AA$ 


\section{The shape and width of lines (8)}

- Beside thermal Doppler broadening there are two other sources of doppler broadening

- Micro-turbulence

- We account for that by an extra doppler velocity $\xi$

$$
\Delta \lambda_{D}=\frac{\lambda}{c} \sqrt{\frac{2 \mathrm{RT}}{\mu}+\xi^{2}}
$$

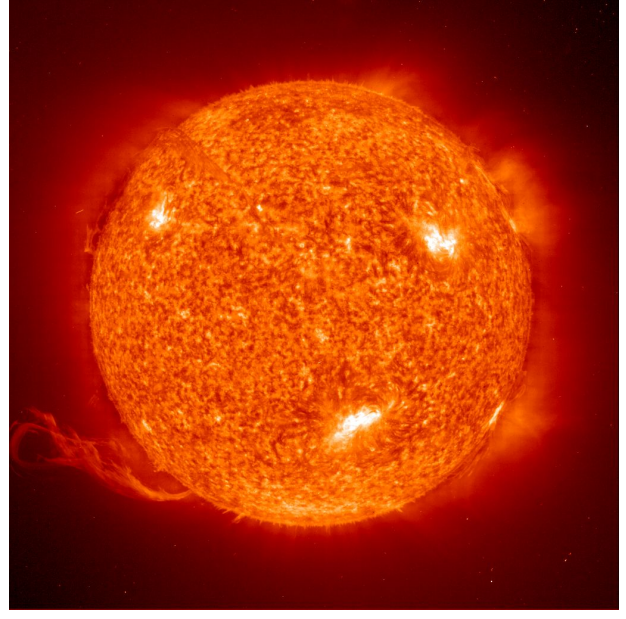

- Stellar rotation

$$
\Delta \lambda= \pm \lambda \frac{v \sin (i)}{c}
$$

Rotational Broadening of Photospheric Absorption Lines

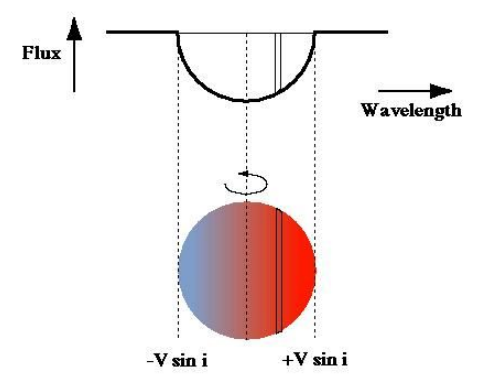




\section{The shape and width of lines (8)}

- Stark broadening

- The absorbing atoms are in an environment where ions and free electrons produce electric fields

- We therefore expect stark splitting of all levels with $I \neq 0$ quantum numbers for elements with hydrogen like configurations.

- Calculating Stark broadening for e.g. Hydrogen is a hard job. Normally we use tabulated data. 


\section{The shape and width of lines (9)}

- Bringing things together

- Up to now we have four major profile types:

- Lorentz profile for damping

- Doppler profile for thermal and turbulent Doppler movement

- Rotational profile

- Stark broadening (for hydrogen like $\mid \neq 0$ species)

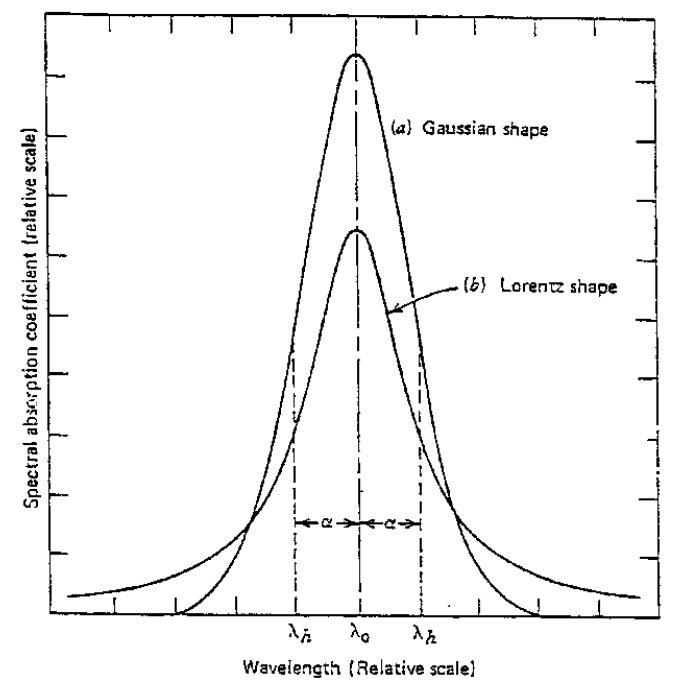




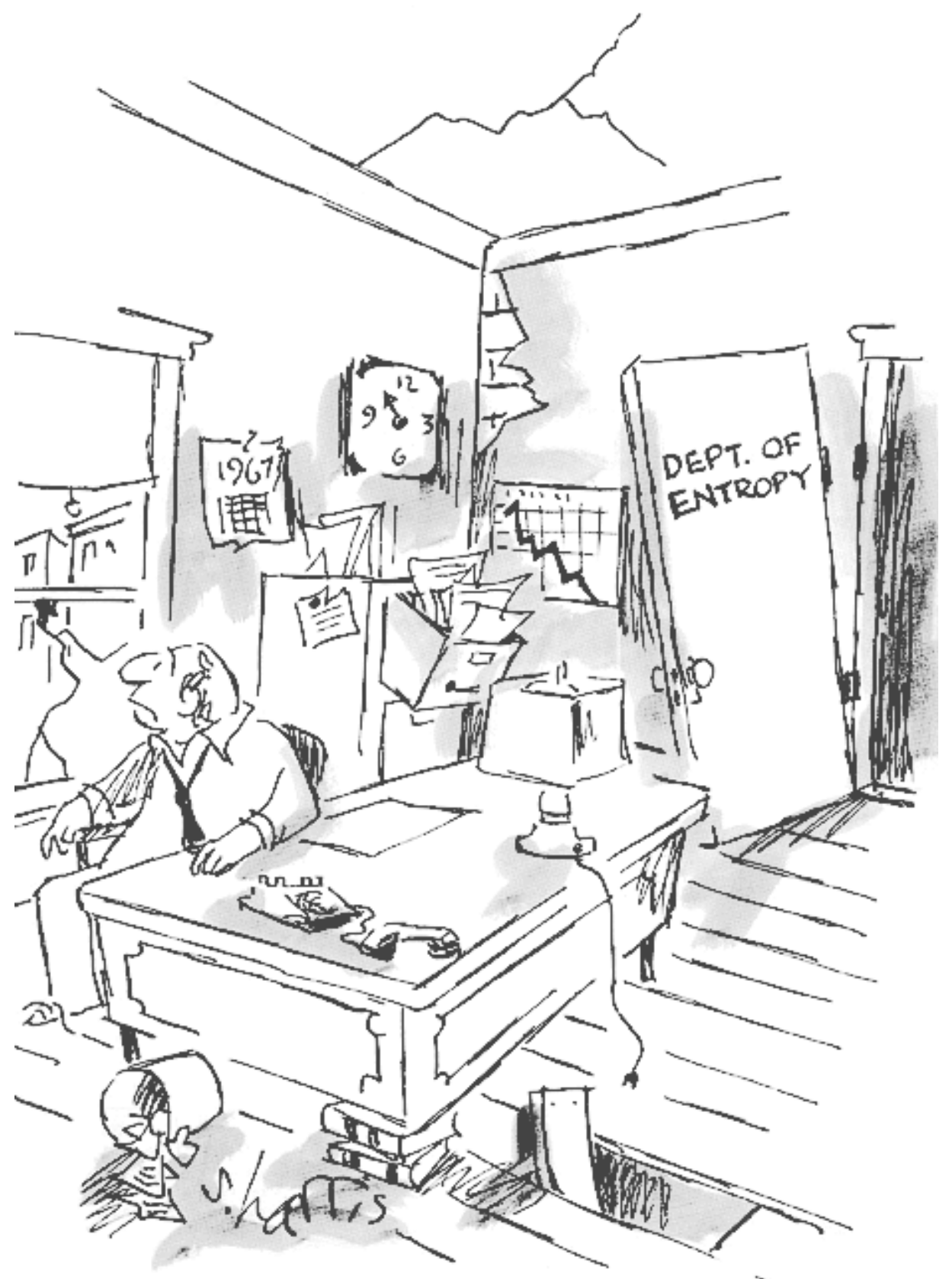




\section{Outline of the lecture}

I. Who I am, what I do.

II.Cool star element abundances

I. Why do we want to know

II.What do we want to know

III.How do we find out

I. The general framework

II.Line formation in model atmospheres

III.The curve of growth

IV.Line fitting

IV.NLTE and why we better use that 


\section{Equivalent width (1)}

- Definition: The equivalent width is the width of a rectangle centered on a spectral line that, on a plot of intensity against wavelength, has the same area as the line.

- By describing a 1.2 line with its EW all individual spectral information is lost

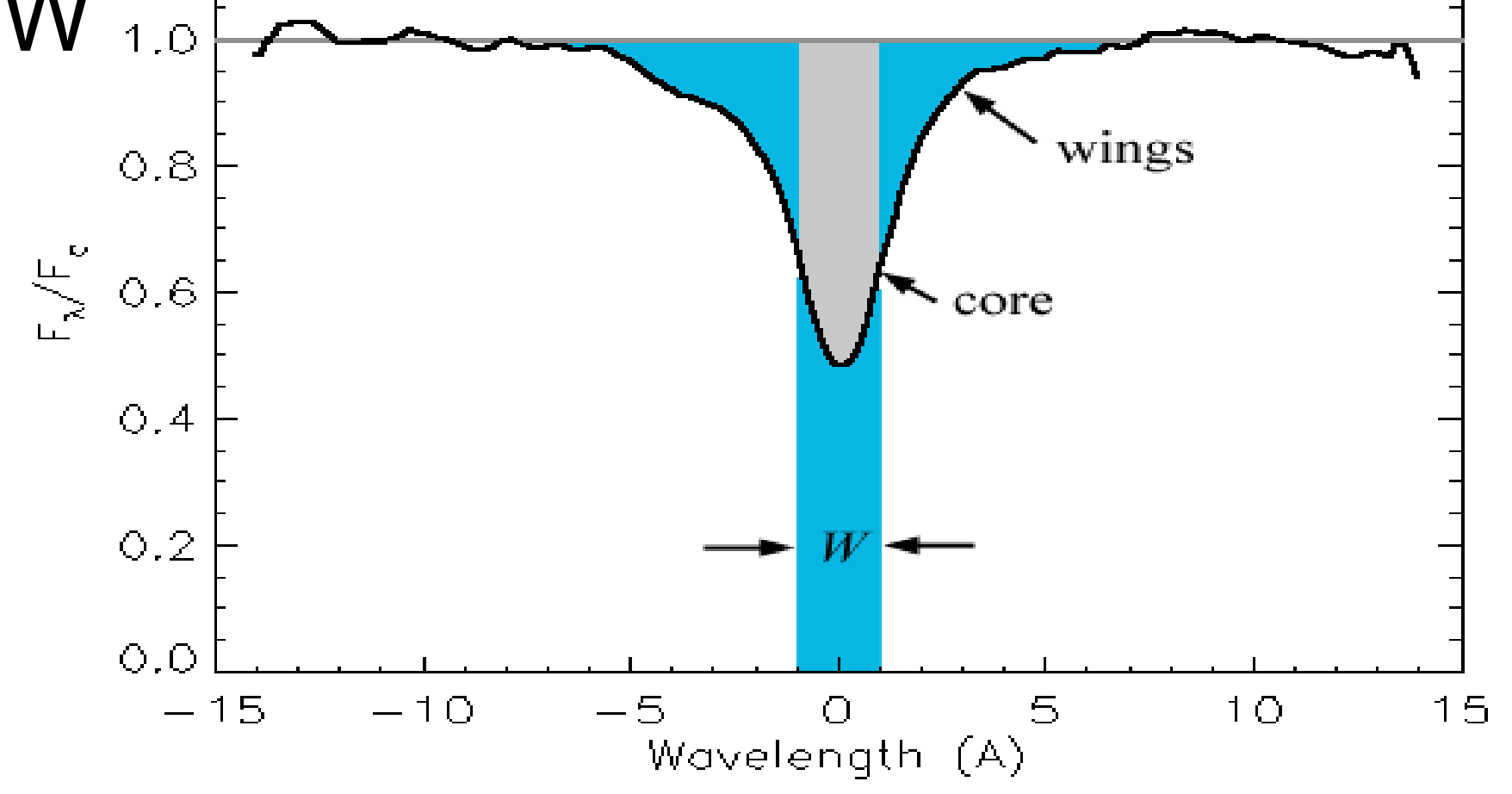




\section{Curve of growth (1)}

- Remember

$$
\begin{aligned}
\frac{I}{I_{0}} & =e^{-\kappa \rho s} \\
& =e^{-\tau}
\end{aligned}
$$

- Where $\rho$ is the density of the absorbers

- If we double the number of atoms absorbing

$\rightarrow$ we double the optical depth

- How does this change the line if we step forward in $T$ 


\section{Curve of growth (2)}

III.III. Curve of growth

- With only Doppler broadening the line evolves like this...

- Showing a Doppler profile

$$
I(\Delta \lambda)=\frac{1}{\sqrt{P i} \Delta \lambda_{D}} e^{-\frac{\Delta \lambda}{\Delta \lambda_{D}}}
$$
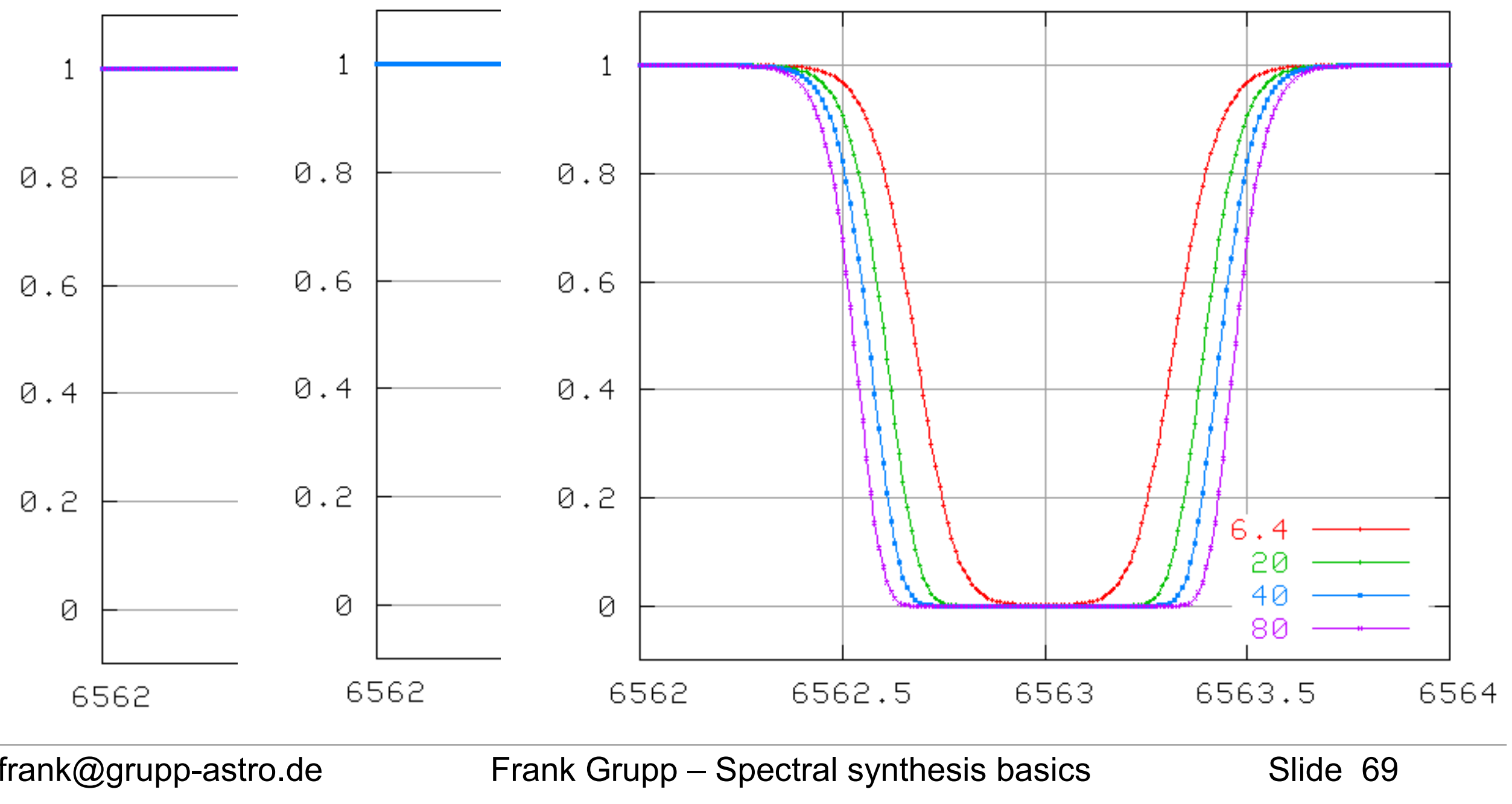


\section{Curve of growth (3)}

- With only Lorentzian profile (pressure damping) things look a bit different

- Showing the profile $\Phi_{r a d}(\Delta \lambda)=\frac{1}{\pi}\left(\frac{a}{\Delta \lambda^{2}+a^{2}}\right) \quad a=\frac{\lambda^{2}}{4 \pi c} \gamma_{r a d}$
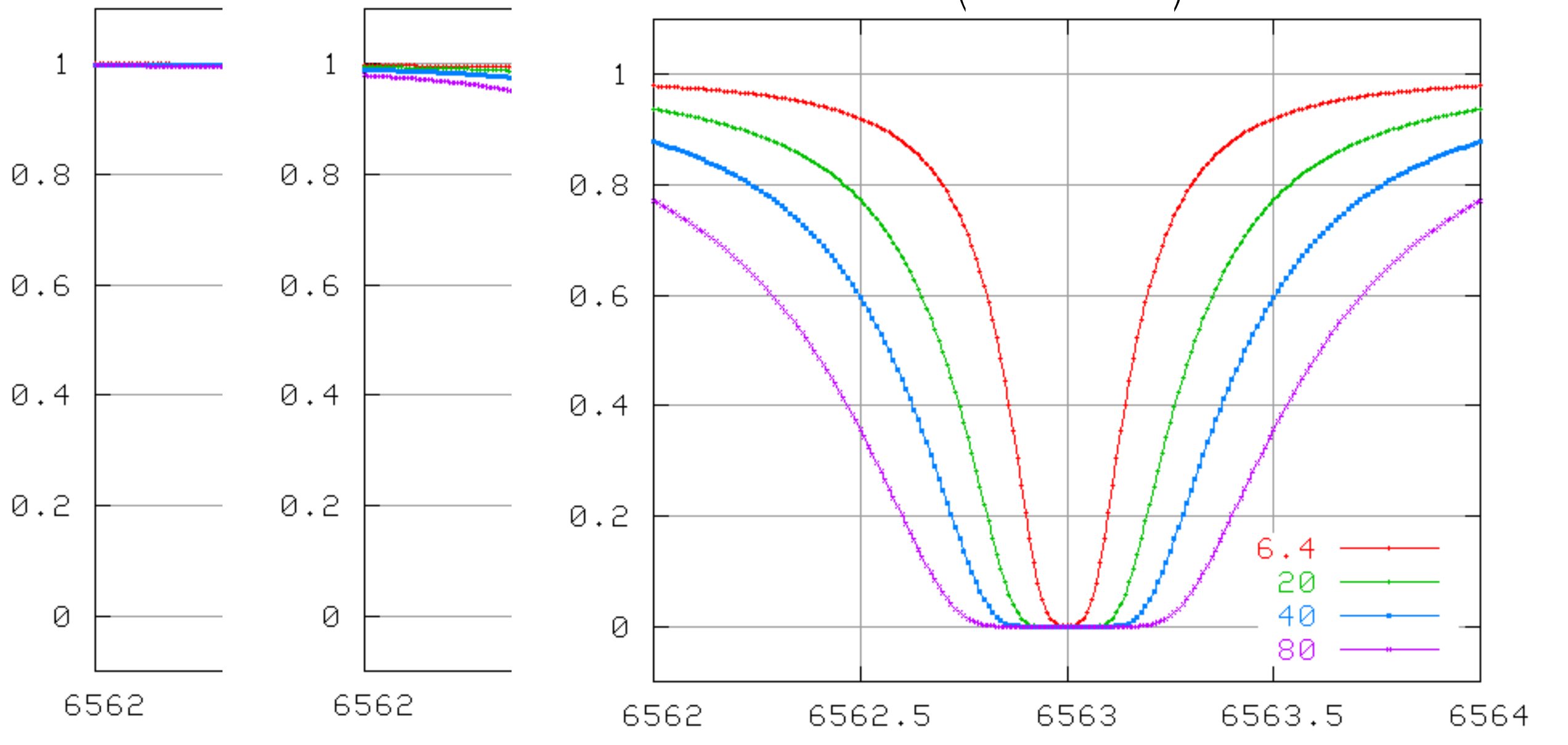


\section{Curve of growth (4)}

- In a direct comparison

- Doppler leads to "boxy" strong lines

- Lorentz to "V-shaped" strong lines

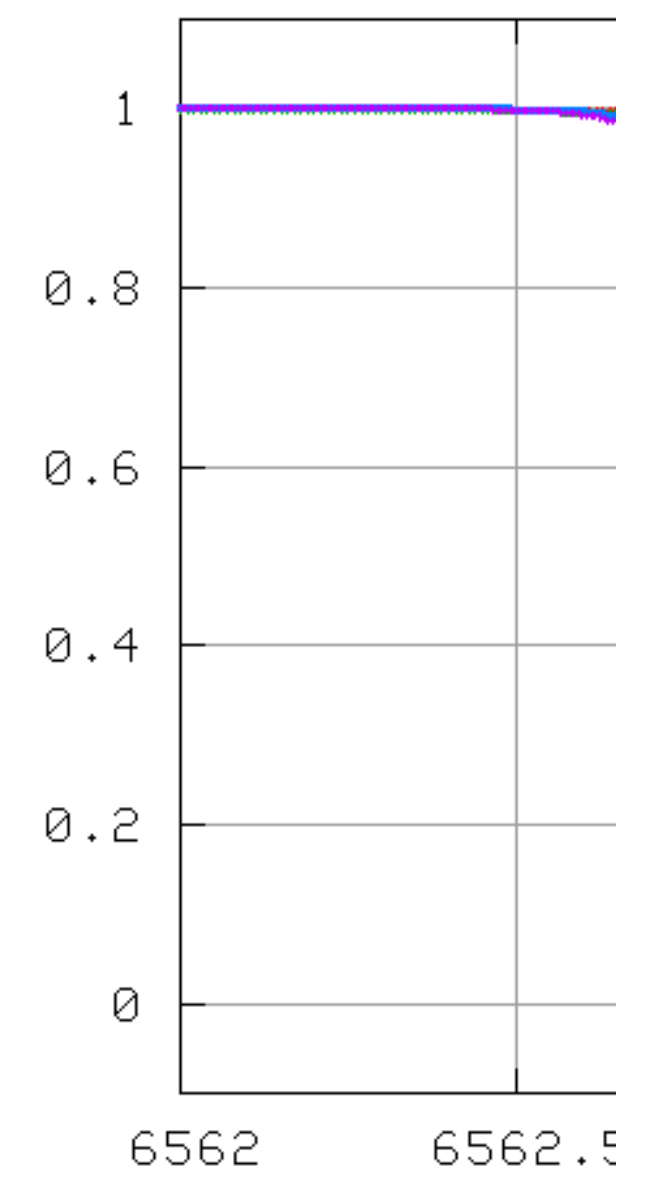

frank@grupp-astro.de

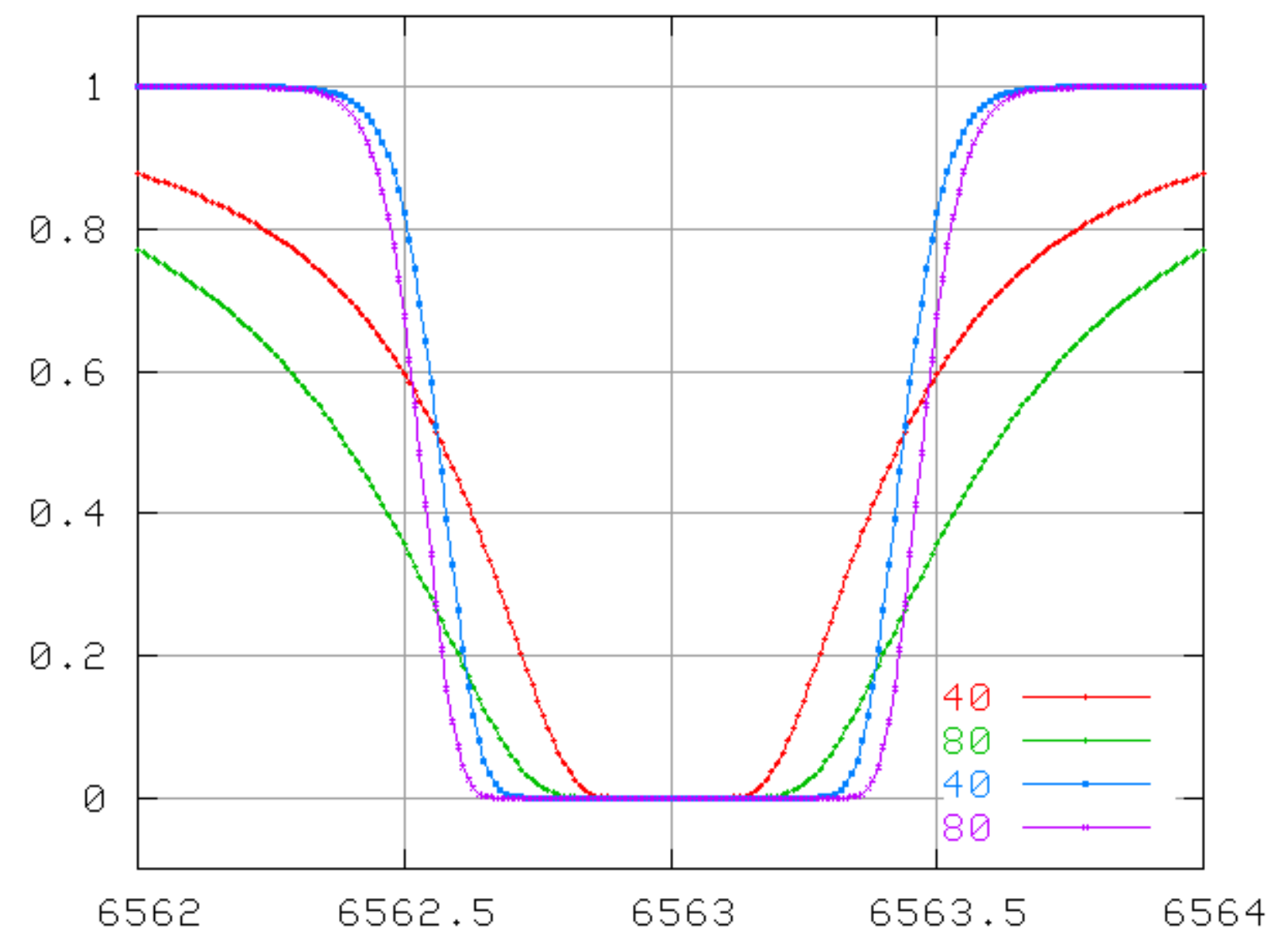

Frank Grupp - Spectral synthesis basics Slide 71 


\section{Curve of growth (4)}

- Both line types together lead to a complex shape

- Lets look at three regimes for this complex line shape:

- Few aborbers: $\quad W \sim N$

- The equivalent with changes linear with the number of absorbers

- Many absorbers, transition region between Doppler and collisional regime:

$$
W \sim \sqrt{\ln (N)}
$$

- Saturated lines, collision dominated

$$
W \sim \sqrt{N}
$$




\section{Curve of growth (5)}

\section{- The curve of growth}

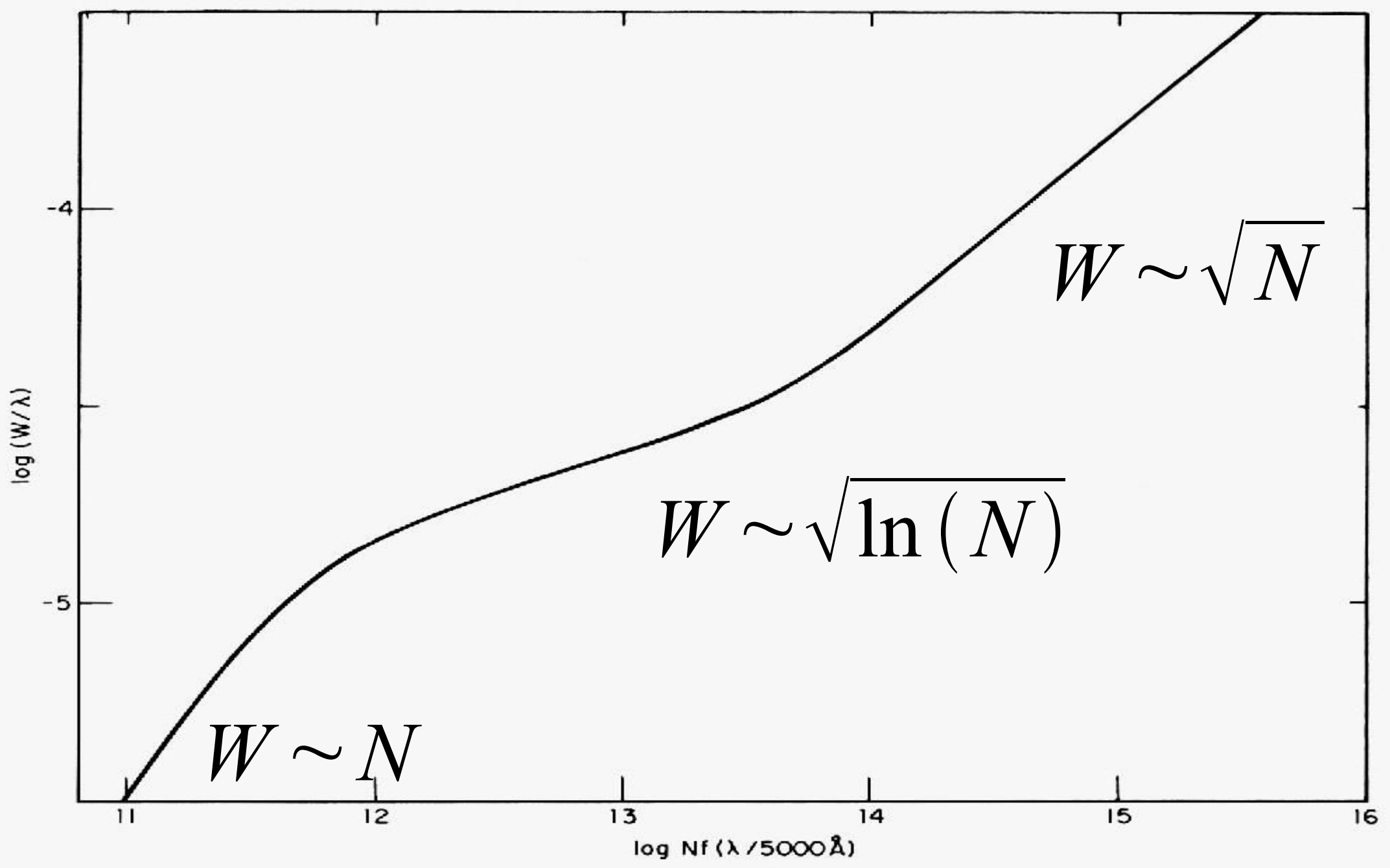

Figure 9.22 A general curve of growth for the Sun. (Figure from Aller, Atoms, Stars, and Nebulae, Revised Edition, Harvard University Press, Cambridge, MA, 1971.) 


\section{Curve of growth (6)}

- For a long time curve of growth analysis have been the primary way to determine element abundances

- Knowing $f, \mathrm{C}_{6}$ and $\lambda$ and measuring $\mathrm{W}$ the only unknown is

$\mathrm{N}$, the number of absorbers

- With better data the method has become outdated

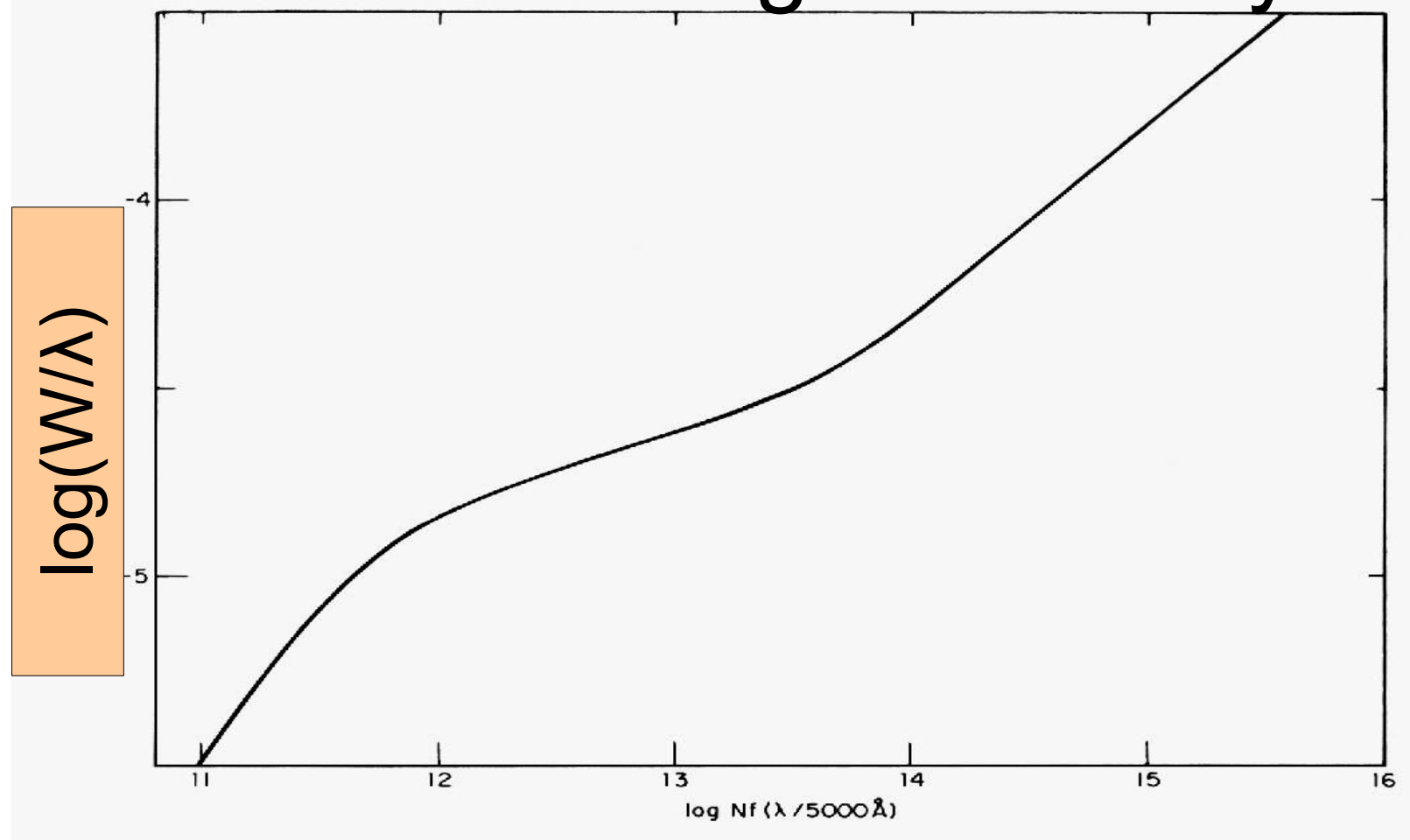

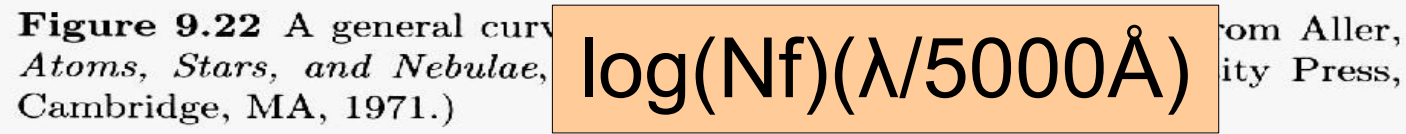




\section{The End}

- And the story goes on....

COSMOLOGY MARCHES ON
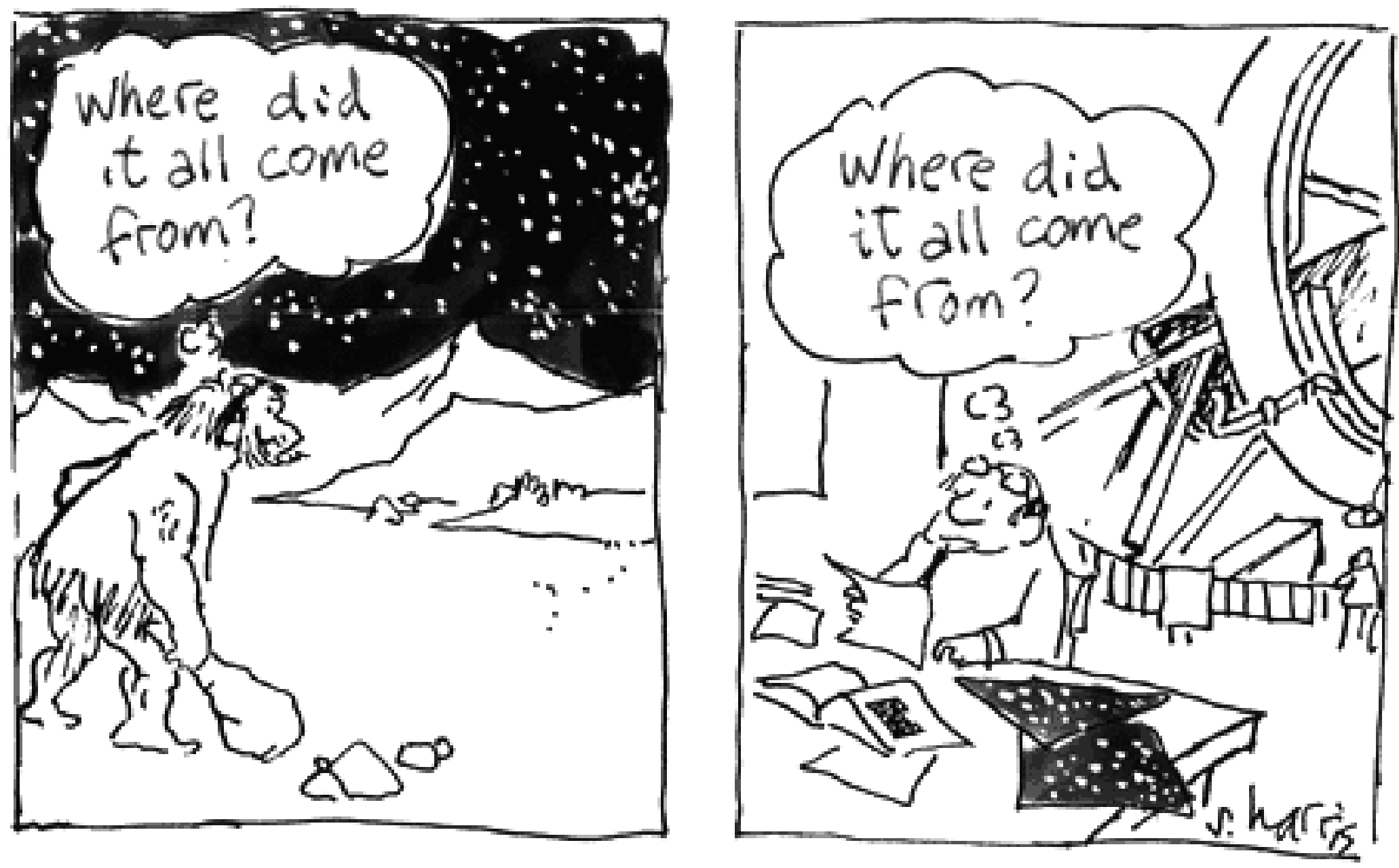